


\section{Tree diversity and carbon stock in various land use systems of Jayapura, Jayawijaya and Merauke Districts, Papua Province}

Subekti Rahayu and Sidiq Pambudi

Working paper no. 268 


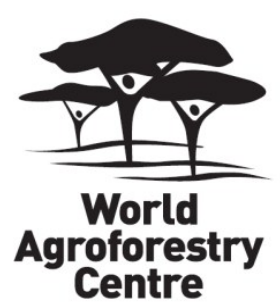

\section{Correct citation}

Rahayu S and Pambudi S. 2017. Tree diversity and carbon stock in various land use systems of Jayapura, Jayawijaya and Merauke Districts, Papua Province. Working Paper 268. Bogor, Indonesia: World Agroforestry Centre (ICRAF) Southeast Asia Regional Program. DOI: http://dx.doi.org/10.5716/WP17359.PDF

Titles in the Working Paper series aim to disseminate interim results on agroforestry research and practices, and stimulate feedback from the scientific community. Other publication series from the World Agroforestry Centre include Technical Manuals, Occasional Papers and the Trees for Change Series.

Published by the World Agroforestry Centre

Southeast Asia Regional Program

JL. CIFOR, Situ Gede, Sindang Barang, Bogor 16680

PO Box 161, Bogor 16001, Indonesia

Tel: +62 2518625415

Fax: +62 2518625416

Email: icraf-indonesia@cgiar.org

ICRAF Southeast Asia website: http://www.worldagroforestry.org/region/southeast-asia/

(C) World Agroforestry Centre 2017

Working paper no. 268

Photos/illustrations: the authors

\section{Disclaimer and copyright}

The views expressed in this publication are those of the author(s) and not necessarily those of the World Agroforestry Centre.

Articles appearing in this publication may be quoted or reproduced without charge, provided the source is acknowledged.

All images remain the sole property of their source and may not be used for any purpose without written permission of the source. 


\section{About the authors}

Subekti Rahayu is a biodiversity and carbon stock specialist at the World Agroforestry Centre. Her main areas of interest include biodiversity conservation, forest and landscape restoration, forest ecology, bioindicators and agroforestry ecology. She is a $\mathrm{PhD}$ candidate on restoration model in Samboja Research Forest, East Kalimantan. She has a master degree in Tropical Biodiversity Conservation from Bogor Agriculture University. She holds a bachelor degree from the same university, majoring in Plant Protection. She has had experience with carbon-stock measurement at plot level since 1998 and delivering training on the subject to various institutions in Indonesia and to local communities in Indonesia and Viet Nam since 2002. Contact: s.rahayu@cgiar.org.

Sidiq Pambudi is research assistant in World Agroforestry Centre Indonesia. His areas of work generally on biodiversity assessment in various land use including analysis vegetation and aboveground carbon stock estimation. Aside from working on field work surveys, analyzing and interpreting data, he has also conducts trainings, and develops reports and briefs. Sidiq also passionate in wildlife study and experienced in conducting fauna inventory in various activity before joining World Agroforestry Centre. He received bachelor degree in Biology from Institut Teknologi Bandung in 2012, focusing on ecology and biosystematics research group. Contact: s.pambudi@cgiar.org 


\begin{abstract}
Various ecosystems, huge area of swampy in Merauke, lowland forest in Jayapura and subalpine forest in Jayawijaya are found in Papua Province. During economic development, most of forest area in Papua is under threat due to source of agriculture land, timber plantation and infrastructure development. Forest conversion various purposes increase carbon emission and biodiversity lost. Developing mitigation action strategies as part of land use planning in Papua was done through considering balance between economic growth and sustainable forest function. Tree diversity and carbon stock is necessarily important to be assessed as input in land use planning. Amount of 64 plots of $20 \mathrm{x} 100 \mathrm{~m}$ in 15 land use systems in Jayapura, Jayawijaya and Merauke were set up to observe tree species diversity and estimate carbon stock in each land use systems. Tree species richness in the natural ecosystems of Jayapura was higher than in Jayawijaya and Merauke due to biophysical conditions. The most important species in the man-mad ecosystems varied depending on the market conditions, with betel nut predominating in Jayapura while the most important was coffee in Jayawijaya and mixed fruits in Merauke. Natural ecosystems provide the best area for tree species conservation, in contrast to man-made ecosystems. Undisturbed dry land forest in Jayapura and Merauke contained the highest carbon stock compared to the other ecosystems, but pines forest and complex agroforest contained the highest carbon stock in Jayawijaya.
\end{abstract}

\title{
Keywords
}

Carbon stock, sustainable development, Papua, tree diversity 


\section{Content}

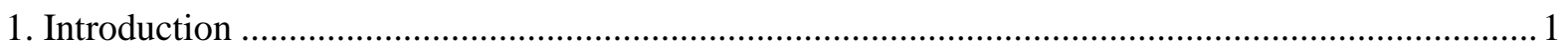

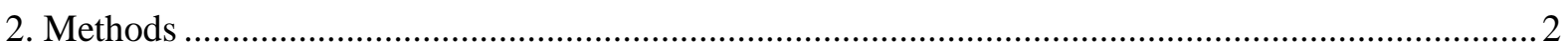

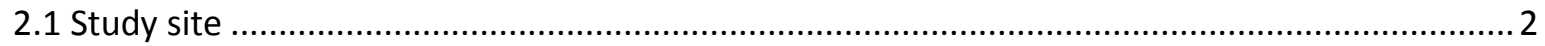

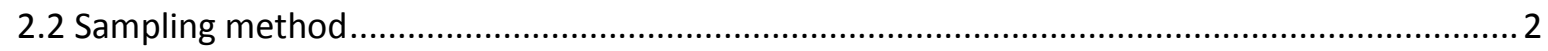

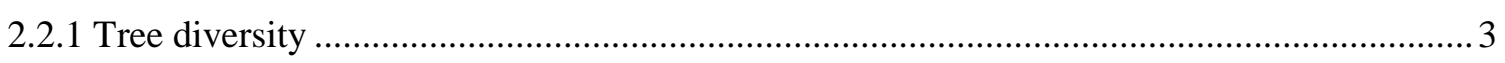

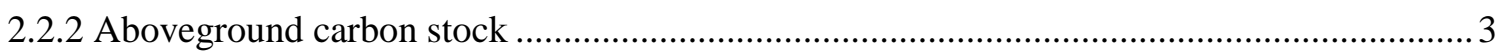

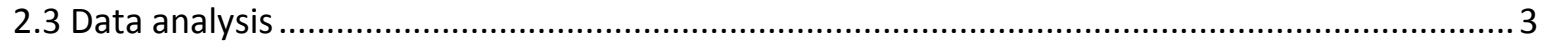

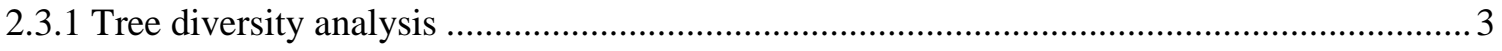

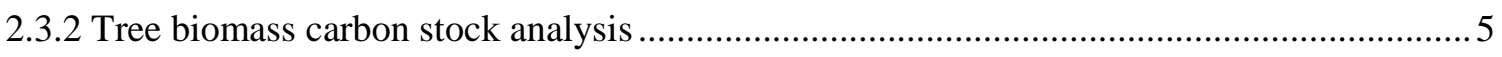

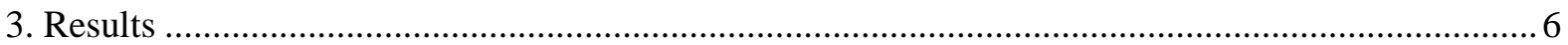

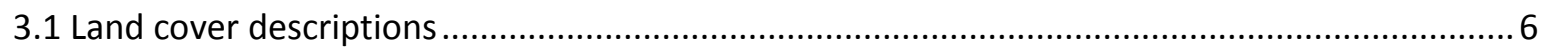

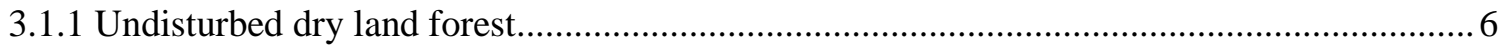

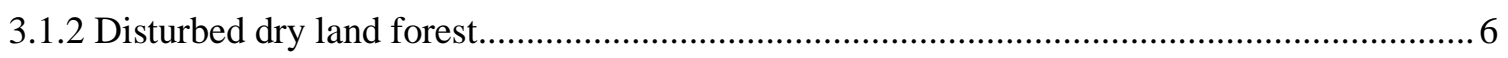

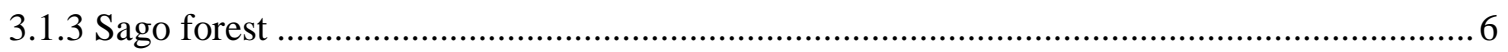

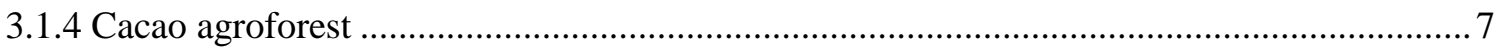

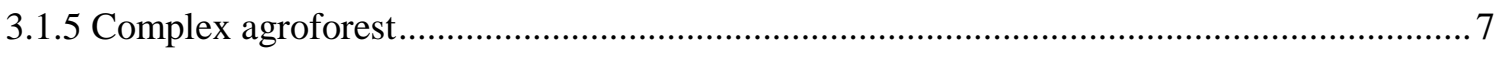

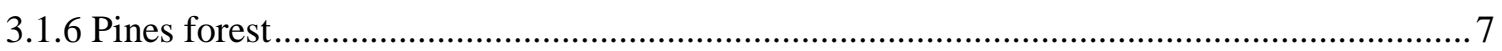

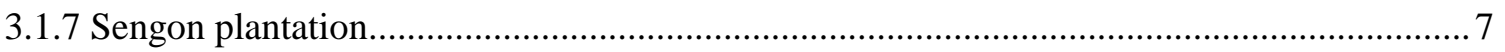

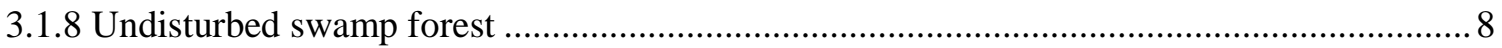

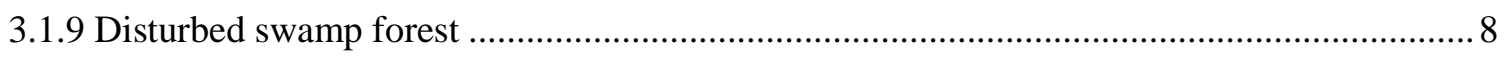

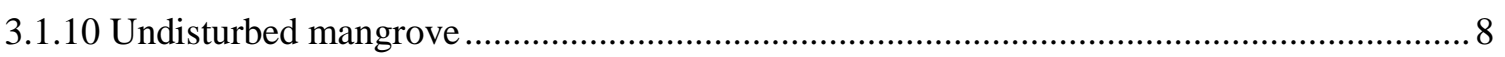

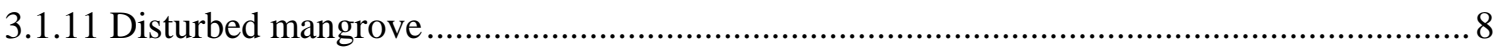

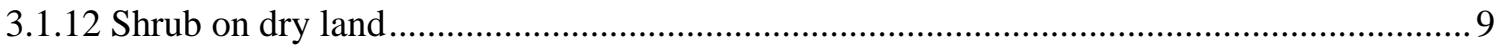

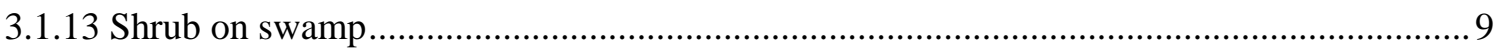

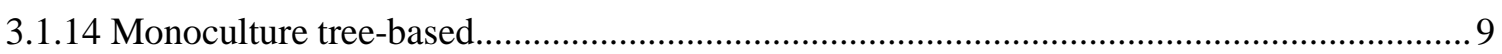

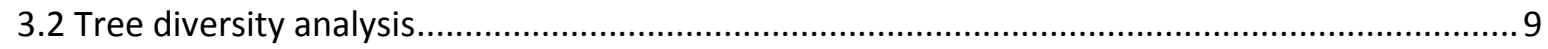

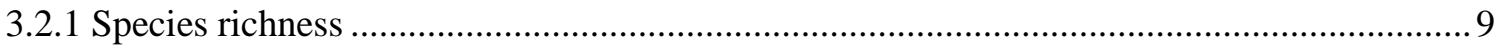

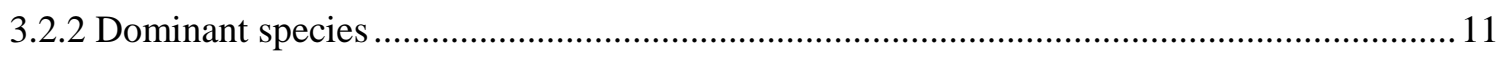

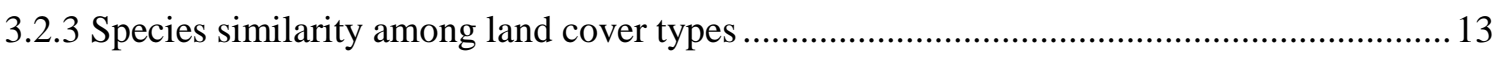

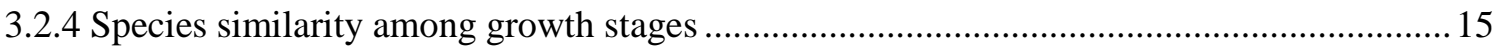

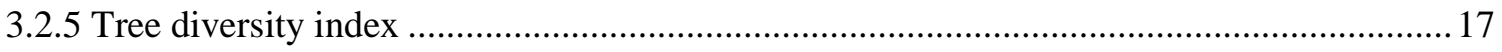

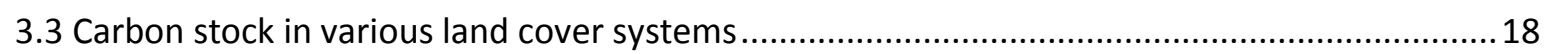

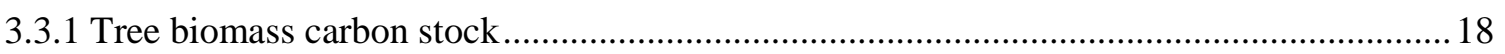

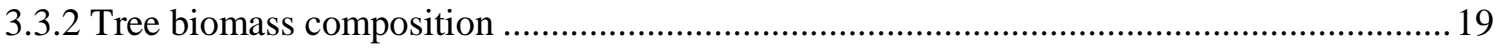

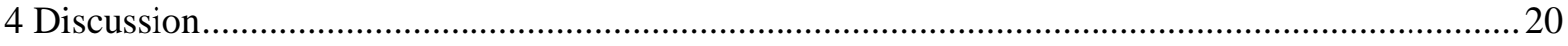

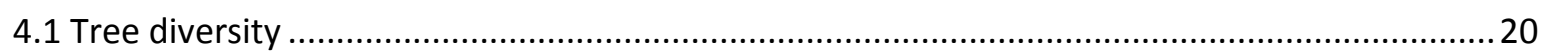

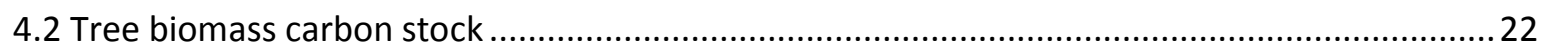


5. Conclusions

6. Suggestion .23

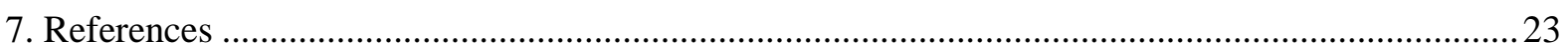

Table Appendix 1. Tree species in each land cover type in Jayapura District.................................25

Table Appendix 2. Tree species in each land cover type in Jayawijaya District ............................ 32

Table Appendix 3. Tree species in each land cover type in Merauke District................................34 


\section{List of Tables}

Table 1. Allometric equations for biomass estimation of specific species .......................................... 5

Table 2. Tree species richness in each land cover systems and each growth stage ............................. 10

Table 3. Three highest IVI tree species in various land cover systems and growth stage vegetation ...11

Table 4. Matrix of species similarity among land cover systems and growth stages in Jayapura ........13

Table 5. Matrix of species similarity among land cover systems and growth stages in Jayawijaya ....13

Table 6. Matrix of species similarity among land cover systems and growth stages in Merauke ........14

Table 7. Species similarity among growth stages in land cover types for Jayapura, Jayawijaya and

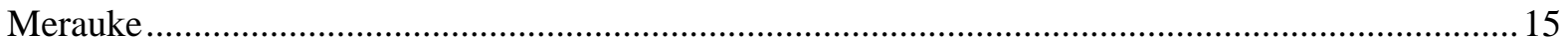

Table 8. Shannon-Wiener diversity in each land covers systems and each growth stage ....................18

Table 9. Plot level aboveground carbon stock in Jayapura, Jayawijaya and Merauke ..........................19

Table 10. Average wood density and tree density by diameter class in various land cover systems

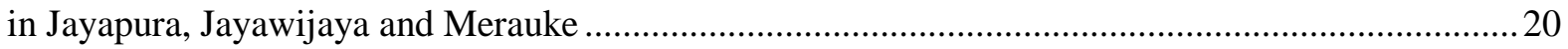

\section{List of Figures}

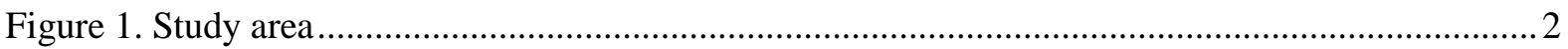

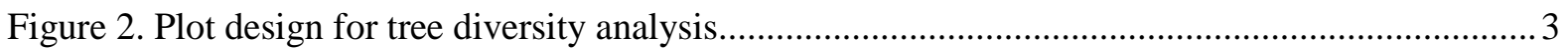

Figure 3. Undisturbed forest of Yepase village ................................................................................. 6

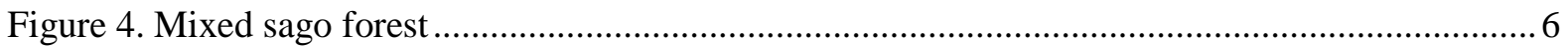

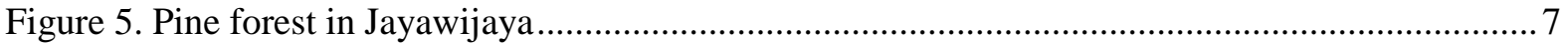

Figure 6. Sengon plantation in Jayawijaya ..................................................................................... 7

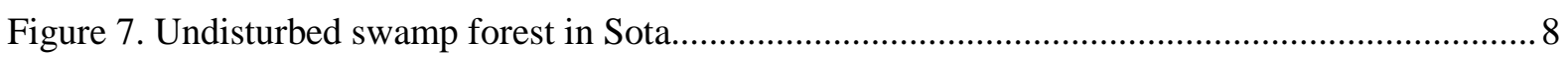

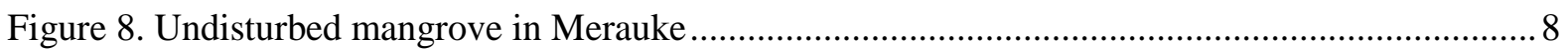

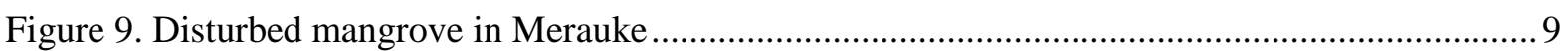

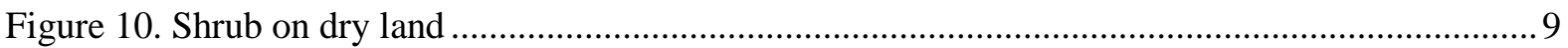





\section{Introduction}

Papua has 25.8 million ha of forest which is equal to $83 \%$ of the province's total area. The most natural forest is in pristine condition which provides significant ecosystem services. Not only does this forest store a vast amount of carbon, therefore contributing to climate regulation, but also it serves as habitat for the extremely rich biodiversity. Many of the species are endemic flora and fauna owing to the diversity of the ecosystems. The topology of natural land cover terrain varies from lowland and montane rainforests, through freshwater swamp forests, mangroves, tropical savanna and grasslands to sub-alpine grasslands.

Greenhouse gases emission in Indonesia mostly is caused by forest conversion into other land uses (Harris et al 2012). The loss of forest cover has led to a loss of biodiversity that reduces the ecosystem services including carbon sequestration. Tree stands are the most affected component, caused by land use changes due to land clearing activity.

Planning in landscape management provides an important means of balancing social-economic needs with environmental sustainability as an action toward climate change mitigation. Therefore, assessment of the characteristics and potential existing land cover is needed in planning future development.

The effort to reduce emissions of $\mathrm{CO}_{2}$ in the atmosphere is resulting in increased carbon sequestration. However, the level of carbon uptake by terrestrial ecosystems is dependent on three aspects: 1) vegetation (species composition, structure, and age of the vegetation), 2) conditions (variations in climate, soils, and natural disturbances such as forest fires) and 3) land management (Hairiah 2007). Of these three aspects, the most appropriate effort to reduce carbon in the air is through the management of vegetation, especially trees, as measuring carbon estimation through trees is relatively easy.

The value of the carbon stock of land cover is highly dependent on the presence of trees, as one component of the carbon pool, with specific information required on tree size, density and the characteristics of each species. While the sustainability of environmental services is not only characterized by the presence of trees alone, the existence of a certain tree species composition can have specific roles including supporting the sustainability of other species. In this study, we assessed the diversity of trees across land use systems and estimated the tree-based carbon stock.

The study location was spread over three districts in Northern Papua (Jayapura), Central Papua (Jayawijaya) and Southern Papua (Merauke) which have specific characteristics of the ecosystems from the central highlands to the dry lowlands and wetlands, different governance systems, a wide range of dynamics and drivers of land-use and land-use changes, and mixed capacity for low emissions development planning and implementation. The objectives of this study were: (1) to estimate the carbon stock in various land use systems as input data for land use planning for low emission development in the LUWES and LUMENS framework and (2) to assess tree diversity in various land use systems as the environmental services component in the LUMENS framework. 


\section{Methods}

\subsection{Study site}

Tree diversity and carbon stock assessment was conducted in three districts of Papua Province, namely,: Jayapura, Jayawijaya and Merauke (Figure 1) The distribution of plot samples in the three districts covered various land use systems, with four types in Jayapura (undisturbed forest, sago forest, cacao agroforest and complex agroforest), five types in Jayawijaya (undisturbed forest, disturbed forest, pines forest, sengon agroforest and complex agroforest), 10 types in Merauke (Undisturbed dry land forest, disturbed dry land forest, undisturbed swamp forest, disturbed swamp forest, undisturbed mangrove forest, disturbed mangrove forest, shrub on dry land, shrub on swamp, complex agroforest , tree-based monoculture plantation).

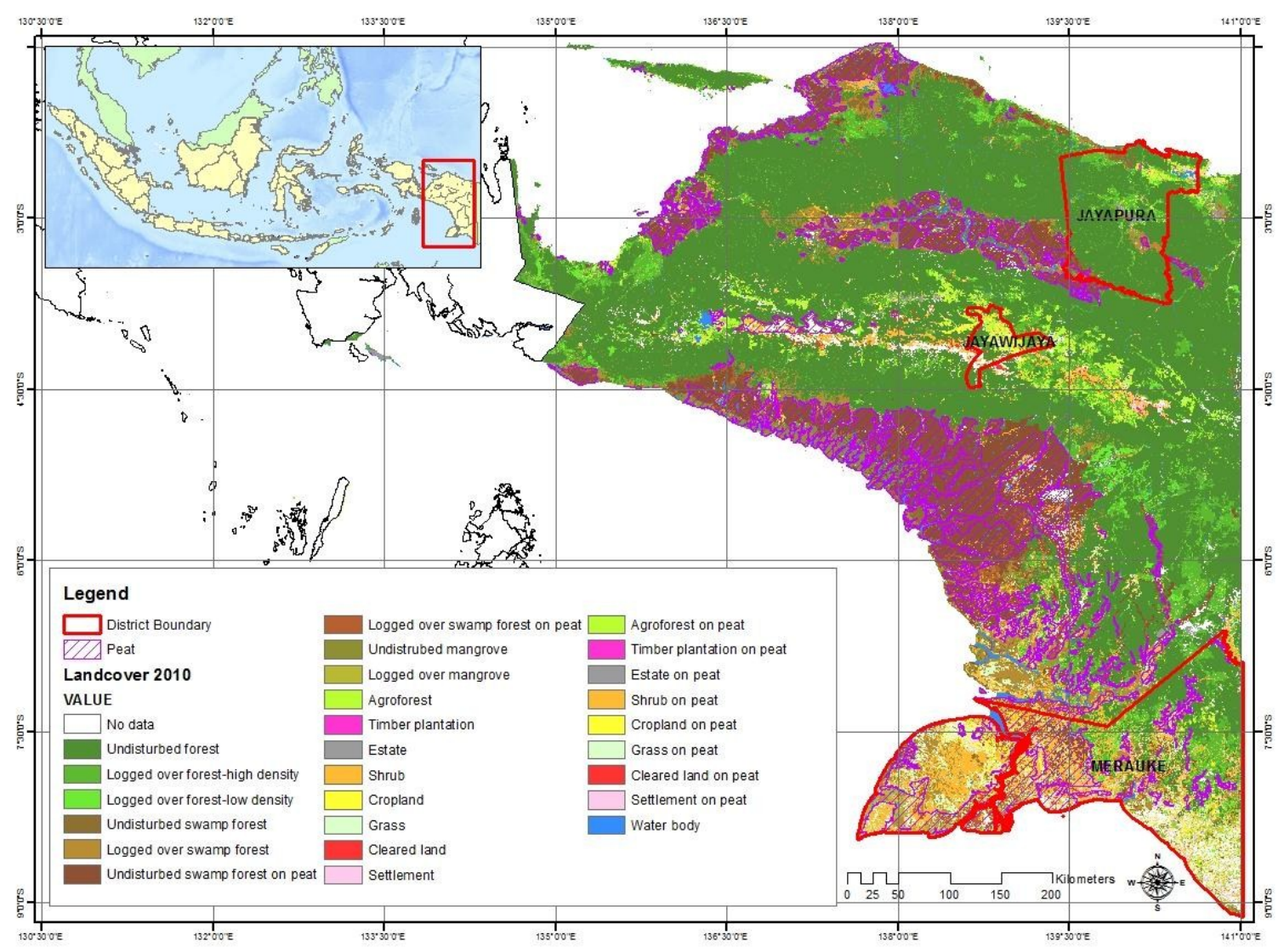

Figure 1. Study area

\subsection{Sampling method}

The sampling method for carbon stock assessment referred to the widespread method developed by Hairiah et al (2011); tree diversity assessment was also undertaken in the same plots as carbon stock determination with small modifications. Plots $2,000 \mathrm{~m}^{2}$ in size $(20 \mathrm{x} 100 \mathrm{~m})$ were placed in middle areas of observed land uses. 


\subsubsection{Tree diversity}

Tree diversity sampling was done for four stages of tree growth, namely: seedling (woody plant less than $2 \mathrm{~m}$ height), sapling (woody plant less than $5 \mathrm{~cm}$ diameter at breast height/DBH, above $2 \mathrm{~m}$ height), pole (woody plant 5-10 cm DBH) and tree (woody plant above $10 \mathrm{~cm} \mathrm{DBH).} \mathrm{Nested} \mathrm{plots}$ were set up in a $20 \times 100$ m plot to observe different stages of vegetative growth (Figure 2), with subplots ( $2 \mathrm{~m}$ x $2 \mathrm{~m}$ for seedling, $5 \mathrm{~m}$ x $5 \mathrm{~m}$ for sapling, $10 \mathrm{~m} \times 10 \mathrm{~m}$ for pole and $20 \mathrm{~m} \times 20 \mathrm{~m}$ for tree). All seedlings and saplings inside the respective subplots were identified through collecting leaf specimens and counting the individual number of each species. All poles and trees inside the respective subplots were measured for $\mathrm{DBH}$ and identified through their leaves, as well as flower and seed specimens if available.

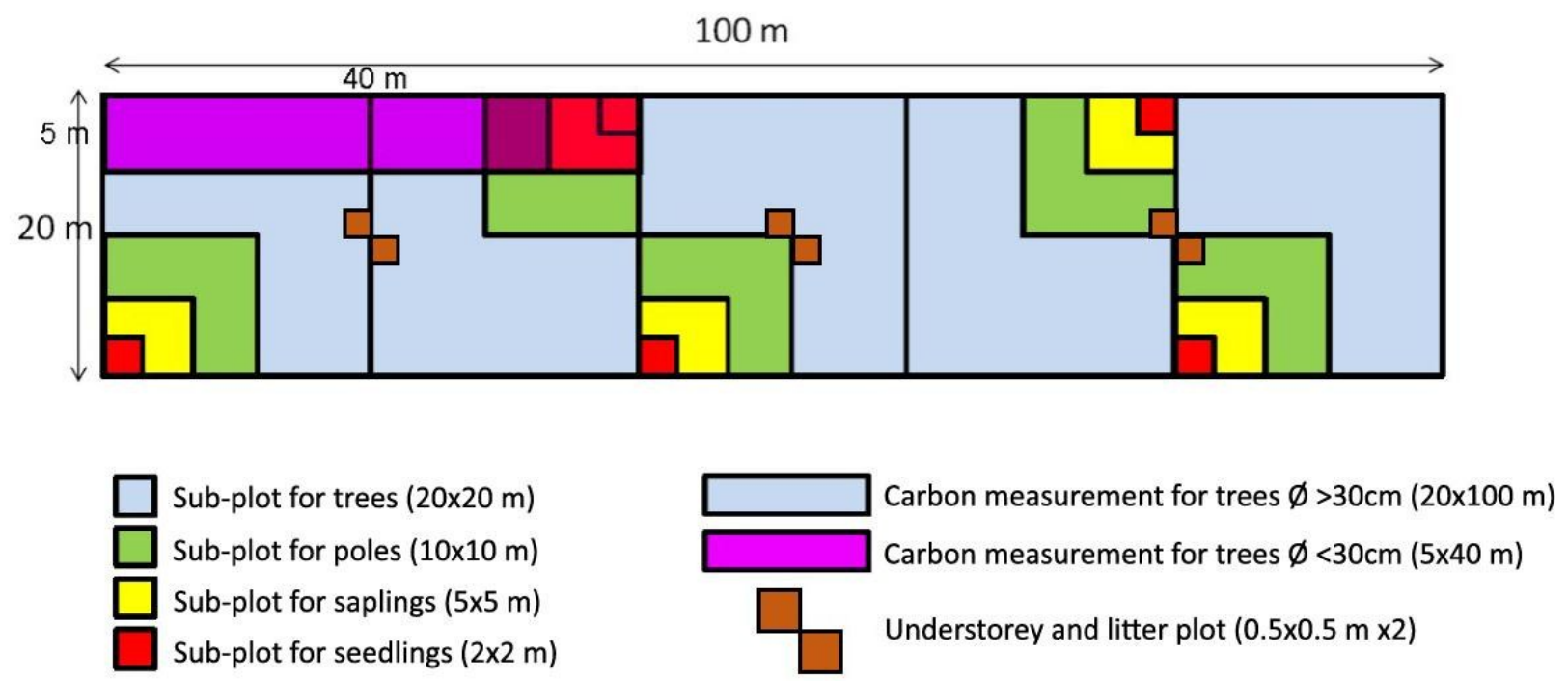

Figure 2. Plot design for tree diversity analysis

\subsubsection{Aboveground carbon stock}

Carbon stock assessment focused on the aboveground vegetation divided into four carbon pools: tree biomass, understorey, tree necromass and litter. A non-destructive method was applied to estimate tree biomass using allometric equations, while understorey and litter sampling used a destructive method by taking samples. Biomass estimation for large living and dead trees above $30 \mathrm{~cm} \mathrm{DBH}$ was analyzed within the 2,000 $\mathrm{m}^{2}$ plot, while the smaller living and dead trees (5-30 $\mathrm{cm} \mathrm{DBH}$ ) were analyzed in a $200 \mathrm{~m}^{2}$ plot (5 m x $40 \mathrm{~m}$ ). Understorey and litter sampling were conducted in a quadrant of $(2 \times 50 \times 50 \mathrm{~cm})$ placed randomly inside the $5 \times 40 \mathrm{~m}$ plot. At least 3 replications were set up during the sampling. All understorey and litter within the quadrant were collected and weighed fresh and then reweighed after oven drying.

\subsection{Data analysis}

\subsubsection{Tree diversity analysis}

Species richness, Important value Index (IVI), Bray-Curtis dissimilarity and Shannon's diversity index were used to analyze tree diversity. Species richness is the number of different species 
represented in an ecological community, landscape or region (Cowell 2009). In this study, species richness represented the number of different species found in a certain land use system.

IVI expresses the dominance of species in the unit area based on their relative frequency, relative density and dominance (Curtis \& McIntosh 1950):

$$
I V I=\text { Relative Frequency }+ \text { Relative Density }+ \text { Relative Dominance }
$$

\section{a. Relative frequency}

Relative frequency is the proportion of quadrats sampled in which the species is represented:

$$
\begin{gathered}
\text { Frequency of species } i=\frac{\text { Number of quadrats with species } i}{\text { Total number of quadrat sampled }} \\
\text { Relative frequency }=\frac{\text { Frequency of species } i}{\text { Total frequency }}
\end{gathered}
$$

\section{b. Relative density}

Relative density is the proportion of a species in relation to the total number of individuals of all species and is estimated by quantifying the number of individuals of a species per unit area:

$$
\begin{gathered}
\text { Density of species } i=\frac{\text { Number of individual species } i}{\text { Area of quadrat sampled }} \\
\text { Relative density }=\frac{\text { Density of species } i}{\text { Total density }}
\end{gathered}
$$

\section{c. Relative dominance}

The dominance of a species is determined by the value of the basal cover. Relative dominance is the coverage value of a species with respect to the sum of the coverage of the rest of the species in the area:

$$
\begin{gathered}
\text { Dominance }(\text { basal area of species } i)=\frac{\pi *(\text { Diameter of species } i)^{2}}{4} \\
\text { Relative dominance }=\frac{\text { Basal area of species } i}{\text { Total basal area }}
\end{gathered}
$$

The similarity index is a comparison of the current vegetation components on an ecological site. A similarity index determines how closely the current plant community resembles either the potential natural community or some other reference community. The similarity index is expressed as a percentage of the reference community that is currently on the ecological site. The similarity index value varies between 0 and 1; 0 indicates very dissimilar and 1 indicates very similar.

Bray-Curtis dissimilarity (B) Index is used to quantify the compositional dissimilarity between two different sites, based on counts at each site. Bray-Curtis dissimilarity Index uses the individual number as the parameter in the calculation, so that both species and individual parameters affect the degree of similarity of two compared sites:

where:

$$
\text { Bray-Curtis similarity }(1-B)=1-\frac{\sum_{i=1}^{S} \mid\left(n_{1 i}-n_{2 i} \mid\right)}{\sum_{i=1}^{S}\left(n_{1 i}+n_{2 i}\right)}
$$


$\mathrm{B}=$ Bray-Curtis dissimilarity Index; $\mathrm{S}=$ total species number in land use 1 and land use 2; $\mathrm{n}_{1}=$ number of individual species $\mathrm{i}$ in land use $1 ; \mathrm{n}_{2}=$ number of individual species $\mathrm{i}$ in land use 2 .

The diversity index is a quantitative measurement that reflects how many different species there are on a site. The Shannon-Wiener diversity index ( $\left.\mathrm{H}^{\prime}\right)$ is a popular index used in ecological studies. It represents the species heterogeneity of a site and incorporates species richness and evenness. The value of H' commonly varies between 0 and3.5, but rarely surpasses 4.5:

where:

$$
H^{\prime}=-\sum p i(\ln p i)
$$

$p i=$ proportion of individual number of each species to total species $\mathrm{i}$

The value of H' represents species heterogeneity and is classified into low $(<1.5)$, medium (1.53.5) and high (> 3.5).

\subsubsection{Tree biomass carbon stock analysis}

The tree biomass carbon stock is generated from individual tree diameter (D) and wood density ( $\square$ using an allometric equation developed by Chave et al (2005) for humid/moist topical forest with precipitation between 1,500 and 4,000 mm/year:

$$
\text { Aboveground Biomass.est }(k g)=\rho * \operatorname{esp}\left(-1.499+2.148 \ln (D)+0.207(\ln (D))^{2}-0.0281(\ln (D))^{3}\right)
$$

For certain species such as coffee, cacao, oil palm, palm, banana and bamboo, we applied specific allometric equations developed by researchers previously (Table 1 ).

\begin{tabular}{|c|c|c|}
\hline Tree species & Allometric Equation & Source \\
\hline Coffee regularly pruned & $(A G B) e s t=0.281 D^{2.06}$ & Arifin 2001 \\
\hline Cacao & $(\mathrm{AGB}) \mathrm{est}=0.1208 \mathrm{D}^{1.98}$ & Yuliasmara 2008 \\
\hline Oil palm & (AGB)est $=0.0976 \mathrm{H}+0.0706$ & ICRAF 2009 \\
\hline Palm & $(A G B) e s t=\exp \{-2.134+2.530 \times \ln (D)\}$ & Brown 1997 \\
\hline Palm & (AGB)est $=4.5+7.7 \times \mathrm{H}$ & Frangi and Lugo 1985 \\
\hline Bamboo & $(\mathrm{AGB}) \mathrm{est}=0.131 \mathrm{D}^{2.28}$ & Priyadarsini 2000 \\
\hline Banana & $(A G B) e s t=0.030 D^{2.13}$ & Arifin 2001 \\
\hline
\end{tabular}

Table 1. Allometric equations for biomass estimation of specific species

The proportion used to convert biomass to carbon is $47 \%$ (Hairiah et al 2011). 


\section{Results}

\subsection{Land cover descriptions}

\subsubsection{Undisturbed dry land forest}

Undisturbed dry land forest in the Jayapura plot samples ranged from low land mixed forest in Yepase (Figure 3) and Harapan village to the higher elevation of Waena village. In Jayawijaya, plot sampling occurred in Wosiala, Gotlan, Sagma II, Sekan and Wililimo villages; while in Merauke, sampling was in three villages of Yagebob, Ulilin, Muting and Eligobel.

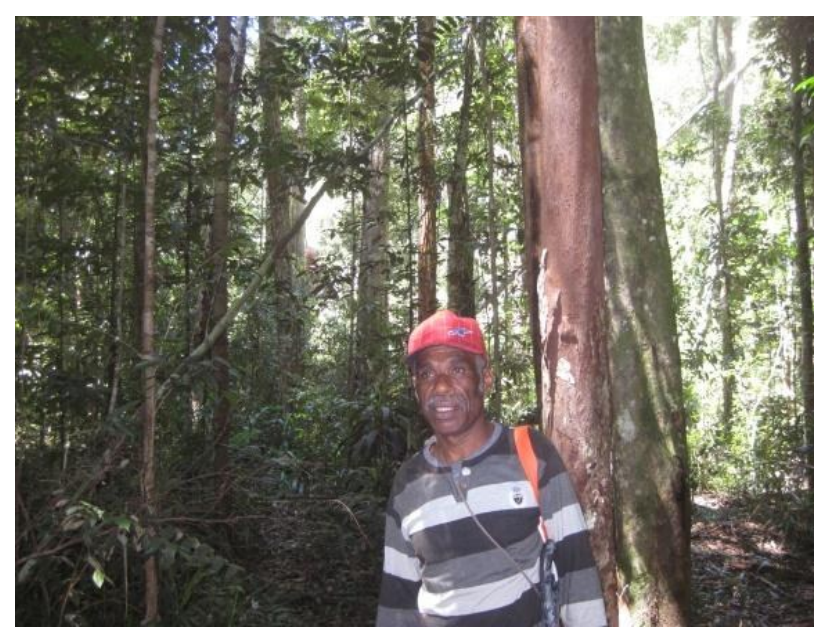

Figure 3. Undisturbed forest of Yepase village

\subsubsection{Disturbed dry land forest}

Disturbed dry land forest plots for Jayawijaya were located in ten villages, they are: Berianggame, Isakusa, Fikha, Molima, Kewir, Napua, Sagma I, Pelima, Ukulik and Wukulik. In Merauke, they were located in four villages of Eligobel, Muting, Bupul and Sota.

\subsubsection{Sago forest}

Sago plot samples for Jayapura District were located in six villages of Folobo, Kehiran, Kenehulu, Kleublow, Loba and Wambena in areas of monoculture and mixed sago forest (Figure 4).

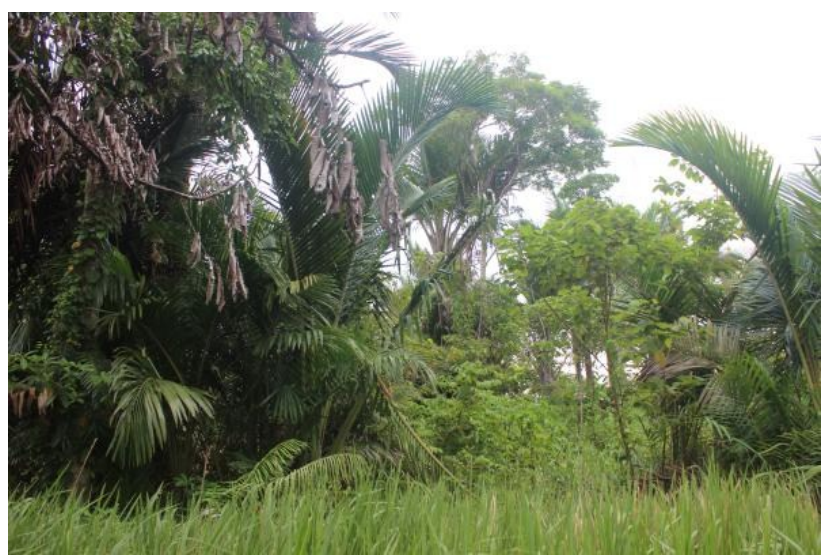

Figure 4. Mixed sago forest 


\subsubsection{Cacao agroforest}

Cacao agroforest plots in Jayapura were located in Sere village. The old growth cacao system are mixed with betel nut and other fruit trees.

\subsubsection{Complex agroforest}

Complex agroforest in Jayapura commonly consists of betel nut and other fruits species, since betel nut is a valued product in Jayapura for traditional consumption. In Jayapura, complex agroforest plots were established in four villages of Wambena, Waibron, Dosai and Sabron Sari. In Jayawijaya, complex agroforest consists of coffee with sengon as a shade species and plots were established in two villages of Jagana and Kepima. In Merauke, complex agroforest consists of various fruit tree species and plots were set up in Muting, Nasai and Tanah Miring.

\subsubsection{Pines forest}

Pine forest is present in Jawajaya as part of mountain forest ecosystems that are dominated by Casuarina papuana and Araucaria cunninghammii (Figure 5).

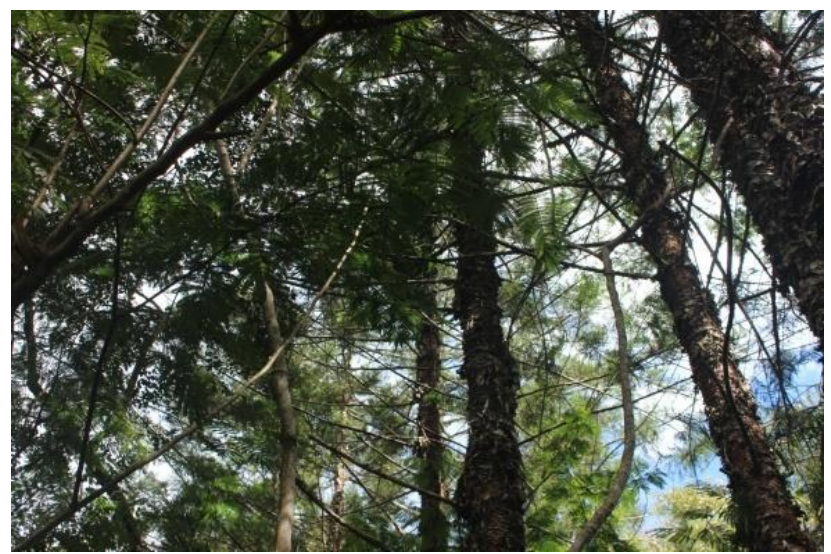

Figure 5. Pine forest in Jayawijaya

\subsubsection{Sengon plantation}

Papua has a familiar clone of sengon that is resistant to rust disease. Large diameter, unlogged sengon trees can be found in Jayawijaya due to the low price of timber (Figure 6). Sengon plots were set up in Tulima.

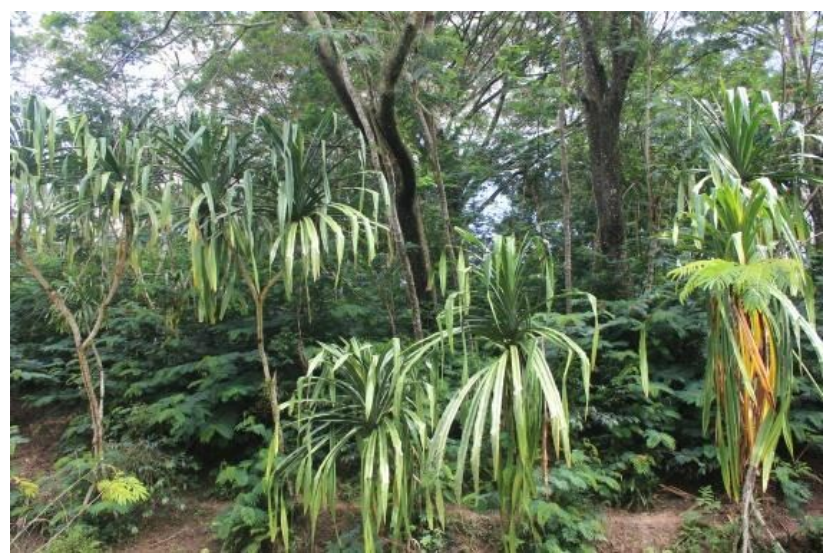

Figure 6. Sengon plantation in Jayawijaya 


\subsubsection{Undisturbed swamp forest}

Undisturbed swamp forest plots were set up in Merauke, located in Malind, Sota and Tanah Miring and were dominated by Acacia crassicarpa, Melaleuca cajuputi and Melaleuca leucodendron (Figure 7).

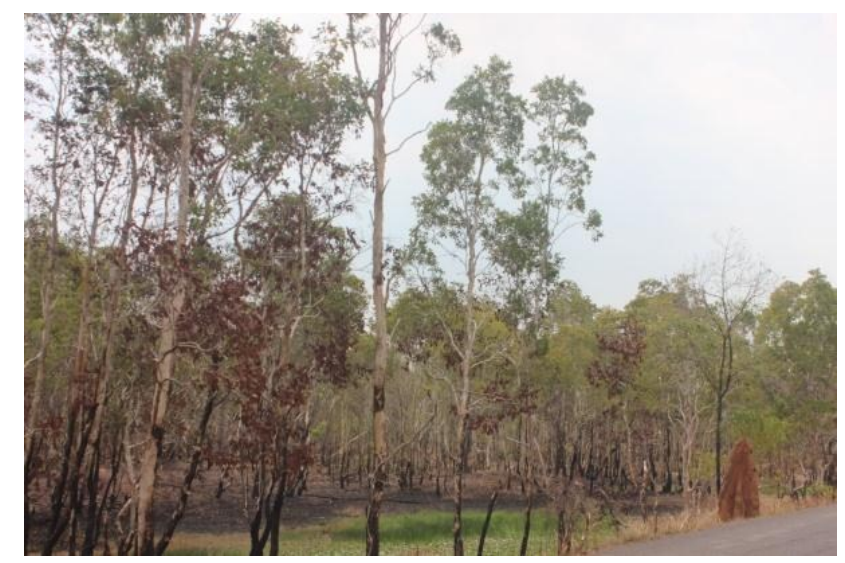

Figure 7. Undisturbed swamp forest in Sota

\subsubsection{Disturbed swamp forest}

Disturbed forest plots were located in Kurik, Sota, Tanah Miring and Merauke. various species were found in disturbed forest, with some plots dominated by Melaleuca cajuputi and Melaleuca leucodendron.

\subsubsection{Undisturbed mangrove}

Undisturbed mangrove plots were set up in Merauke and Semangga and contained various mangrove species (Figure 8).

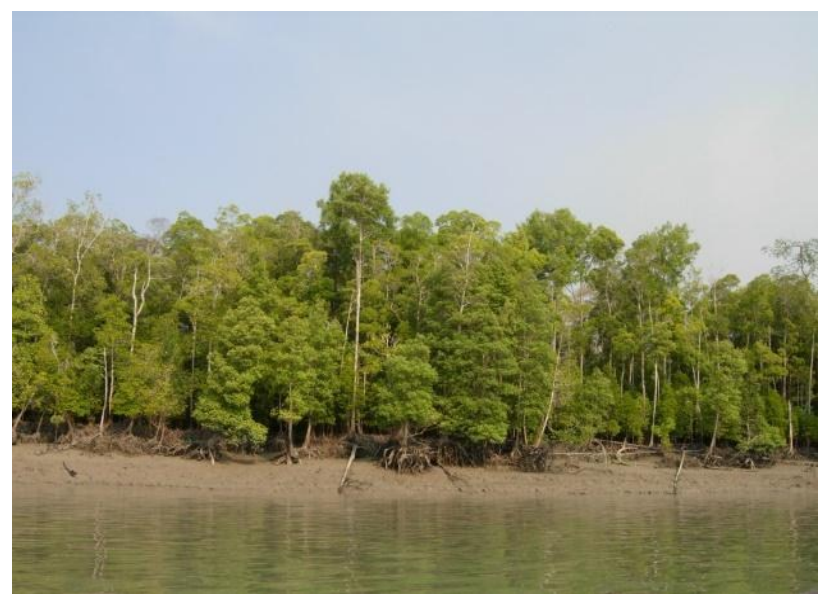

Figure 8. Undisturbed mangrove in Merauke

\subsubsection{Disturbed mangrove}

Disturbed mangrove plots were set up in Malind, Semangga and Merauke. The species composition in disturbed mangrove is similar to undisturbed mangrove, but they have different population densities (Figure 9). 


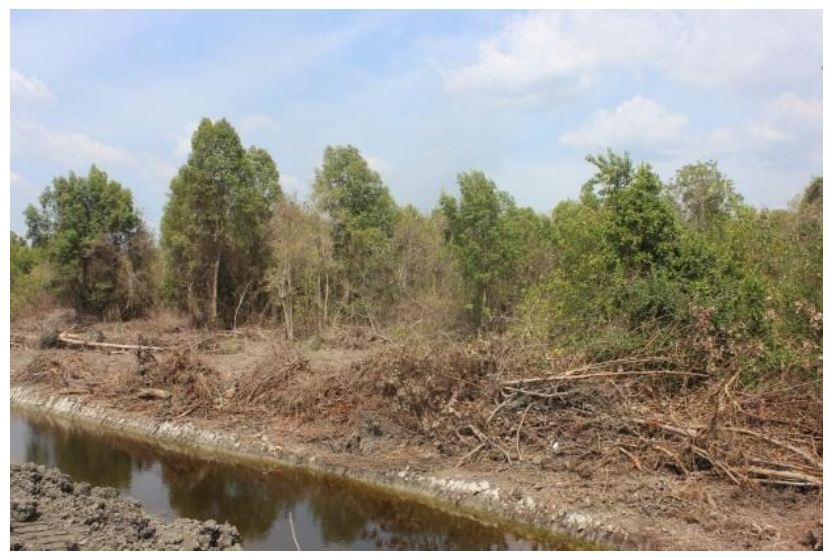

Figure 9. Disturbed mangrove in Merauke

\subsubsection{Shrub on dry land}

Shrubs commonly grow as natural regeneration from forest after disturbance from fire or logging activity. Late succession and pioneer species are found in shrub land cover (Figure 10). Shrub plot samples were set up in Sota, Tanah Miring and Jagebob.

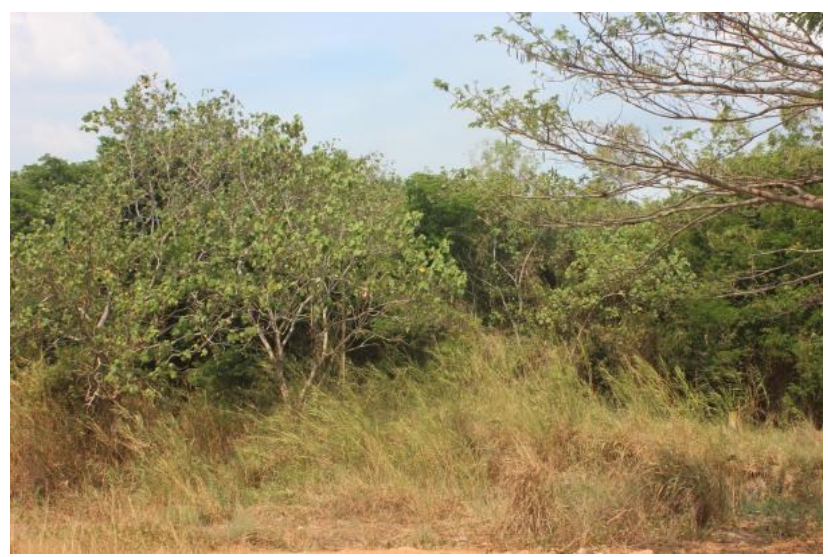

Figure 10. Shrub on dry land

\subsubsection{Shrub on swamp}

Similar to shrub on dry land, there were late succession and pioneer species associated with the swamp ecosystem found in the plots located in Sota, Tanah Miring, Semangga and Kurik.

\subsubsection{Monoculture tree-based}

Tree-base monoculture systems in Merauke consist of cashew in Jagebob, rambutan in Ulilin and rubber in Muting.

\subsection{Tree diversity analysis}

\subsubsection{Species richness}

Species richness in the three districts of Papua as a measure of the different ecological condition of low dry land (Jayapura), highland (Jayawijaya) and low wet land (Merauke) was significantly different (Table 2). Undisturbed forest, representing natural ecosystems in Jayapura, contained the highest number of tree species compared to Jayawijaya and Merauke. Disturbing the forest ecosystems, in both Jayapura and Merauke did not have much effect on tree species richness, since the total species richness between undisturbed and disturbed forest was relatively similar. However, 
further analysis of similar species among different land cover types and among the vegetative growth stages is needed to quantify the level of disturbance and the regeneration process after disturbance.

Species richness of seedlings and saplings in undisturbed dry land forest in Jayapura was similar to the tree stage, but different for poles that were only about $50 \%$ of the tree stage. A low number of overlapping species among growth stages in the undisturbed dry land forest of Jayapura indicated high species diversity. In Jayawijaya and Merauke, species richness decreased with the vegetative growth stage and there were a large number of overlapping species among growth stages.

Disturbed dry land forests in Jayawijaya contained lower species richness than Merauke, with 51 species found in 11 plots. The species richness in the undisturbed and disturbed dry land forest of Merauke was higher than in swamp and mangrove. Complex agroforest in Jayapura had a similar species richness to Merauke, but was higher than for Jayawijaya.

Comparing the total species richness in each land cover system, species richness in Jayapura was in balance for all growth stages, at about $40 \%$ in undisturbed dry land forest, but $50 \%$ and $100 \%$ contributed by the tree stage for sago and cacao agroforest, respectively. In Jayawijaya, the contribution of species richness in each growth stage was higher than for Jayapura at about $60 \%$, with Merauke the highest at $70 \%$. There was a similar trend in the species richness composition for total species in complex agroforest in the three districts, although Jayawijaya did have lower species richness.

Table 2. Tree species richness in each land cover systems and each growth stage

\begin{tabular}{|c|c|c|c|c|c|c|c|}
\hline District & Land cover & No. of plot & Seedling & Sapling & Pole & Tree & Total species \\
\hline \multirow[t]{4}{*}{ Jayapura } & Undisturbed dry land forest & 5 & 82 & 72 & 48 & 83 & 183 \\
\hline & Sago forest & 3 & 9 & 9 & 6 & 15 & 26 \\
\hline & Cacao agroforest & 2 & - & - & 4 & 22 & 22 \\
\hline & Complex agroforest & 6 & 21 & 20 & 16 & 20 & 45 \\
\hline \multirow[t]{5}{*}{ Jayawijaya } & Undisturbed dry land forest & 6 & 30 & 26 & 28 & 34 & 49 \\
\hline & Disturbed dry land forest & 11 & 27 & 30 & 23 & 35 & 51 \\
\hline & Pine forest & 1 & 3 & 6 & 5 & 4 & 10 \\
\hline & Sengon plantation & 1 & 2 & 2 & 1 & 2 & 3 \\
\hline & Complex agroforest & 2 & 8 & 7 & 7 & 12 & 18 \\
\hline \multirow[t]{10}{*}{ Merauke } & Undisturbed dry land forest & 4 & 32 & 40 & 58 & 60 & 64 \\
\hline & Disturbed dry land forest & 4 & 46 & 43 & 48 & 47 & 62 \\
\hline & Undisturbed swamp forest & 3 & 28 & 35 & 28 & 23 & 38 \\
\hline & Disturbed swamp forest & 4 & 29 & 29 & 34 & 27 & 41 \\
\hline & Undisturbed mangrove & 3 & 17 & 16 & 14 & 16 & 18 \\
\hline & Disturbed mangrove & 3 & 10 & 11 & 12 & 14 & 14 \\
\hline & Shrub on dry land & 3 & 21 & 16 & 15 & 26 & 27 \\
\hline & Shrub on swamp & 4 & 22 & 20 & 27 & 26 & 32 \\
\hline & Complex agroforest & 3 & 17 & 29 & 16 & 18 & 45 \\
\hline & Tree-based monoculture plantatior & 3 & 5 & 1 & 4 & 4 & 8 \\
\hline
\end{tabular}




\subsubsection{Dominant species}

The Important Value Index (IVI) expresses the dominance of species in the sampled area. The species with the highest IVI were different among the types of land cover in the three districts of Jayapura, Jayawijaya and Merauke and among growth stages (Table 3). In this study, only the three highest IVI values have been recorded for each land cover for all growth stages of vegetation.

The dominant species differed in undisturbed dry land forest in each of Jayapura, Jayawijaya and Merauke. The species dominant in Jayapura and Merauke were typically low land mixed forest species, whereas Jayawijaya was dominated by mountainous species. Other natural ecosystems (such as disturbed forest in dry land, swamp and mangrove, as well as shrub in dry land and mangrove) were dominated by naturally growing species. However, the dominant species in the man-made ecosystems (such as cacao agroforest, complex agroforest and monoculture tree-based) were affected by human preference. Betel nut (Areca catechu) and cacao (Theobroma cacao) were the most dominant pole species in Jayapura, while coconut (Cocos nucifera) and matoa (Pometia pinnata) were the most dominant in the tree stage.

Ficus microcarpa was the second most dominant species after Paraserianthes falcataria in the tree stage in the coffee-based agroforest system in Jayawijaya indicating natural regeneration of the species due to low management. In sago forest, sago (Metroxylon sago) was the most dominant species in almost every vegetative growth stage, except for seedlings, as sago seedlings were in a clump included in the 5 x $5 \mathrm{~m}$ plot. Macaranga hypoleuca, a pioneer species, was the second most dominant in the sago forest.

Avicennia eucalyptifolia is mangrove species that is consistently included in the three most dominant species in all growth stages in undisturbed and disturbed mangrove in Merauke. Even though Rhizophora was the most dominant species in all growth stages in undisturbed mangrove, Acacia eucaluptifolia was the most dominant in disturbed mangrove. Melaleuca leucadendra was dominant species in undisturbed swamp forest in all growth stages and was the most dominant species in the pole and tree stages. Even in disturbed swamp forest, Melaleuca leucadendra was the most dominant in the tree stage. The dominance of Acacia increased in disturbed swamp forest in the sapling, seedling and tree stages compared to undisturbed swamp forest.

Table 3. Three highest IVI tree species in various land cover systems and growth stage vegetation

\begin{tabular}{|c|c|c|c|c|}
\hline Land cover & Seedling & Sapling & Pole & Tree \\
\hline \multicolumn{5}{|c|}{ Jayapura } \\
\hline $\begin{array}{l}\text { Undisturbed dry } \\
\text { land forest }\end{array}$ & $\begin{array}{l}\text { Syzygium naiadum } \\
\text { Maranthes } \\
\text { corymbosa } \\
\text { Haplolobus } \\
\text { floribundus }\end{array}$ & $\begin{array}{l}\text { S. naiadum } \\
\text { H. floribundus } \\
\text { Ternstroemia merrilliana }\end{array}$ & $\begin{array}{l}\text { Syzygium cf } \\
\text { decipiens } \\
\text { Canarium littorale } \\
\text { Cryptocarya sp. }\end{array}$ & $\begin{array}{l}\text { Syzygium decipiens } \\
\text { Agathis sp. } \\
\text { H. floribundus }\end{array}$ \\
\hline Sago forest & $\begin{array}{l}\text { Pometia pinnata } \\
\text { Myristica subaculata } \\
\text { Macaranga } \\
\text { hypoleuca }\end{array}$ & $\begin{array}{l}\text { Metroxylon sago } \\
\text { M. hypoleuca } \\
\text { Theobroma cacao }\end{array}$ & $\begin{array}{l}\text { M. sago } \\
\text { Areca catechu } \\
\text { M. hypoleuca }\end{array}$ & $\begin{array}{l}\text { M. sago } \\
\text { M. hypoleuca } \\
\text { A. catechu }\end{array}$ \\
\hline Cacao agroforest & - & - & $\begin{array}{l}\text { T. cacao } \\
\text { A. catechu } \\
\text { Nephelium lappaceum }\end{array}$ & $\begin{array}{l}\text { A. catechu } \\
\text { N. lappaceum } \\
\text { T. cacao }\end{array}$ \\
\hline
\end{tabular}




\begin{tabular}{|c|c|c|c|c|}
\hline Land cover & Seedling & Sapling & Pole & Tree \\
\hline Complex agroforest & $\begin{array}{l}\text { A. catechu } \\
\text { Ficus sp. } \\
\text { Hibiscus sp. }\end{array}$ & $\begin{array}{l}\text { Sterculia ceramica } \\
\text { P. pinnata } \\
\text { A. cathechu }\end{array}$ & $\begin{array}{l}\text { A. catechu } \\
\text { T. cacao } \\
\text { N. lappaceum }\end{array}$ & $\begin{array}{l}\text { Cocos nucifera } \\
P . \text { pinnata } \\
\text { N. lappaceum }\end{array}$ \\
\hline \multicolumn{5}{|c|}{ Jayawijaya } \\
\hline $\begin{array}{l}\text { Undisturbed dry land } \\
\text { forest }\end{array}$ & $\begin{array}{l}\text { Araucaria } \\
\text { cunninghamii } \\
\text { Ficus microcarpa } \\
\text { Meliosma angulata }\end{array}$ & $\begin{array}{l}\text { Glochidion sp. } \\
\text { Mallotus sp. } \\
\text { A. cunninghamii }\end{array}$ & $\begin{array}{l}\text { F. microcarpa } \\
\text { A. cuninghamii } \\
\text { Glochidion sp. }\end{array}$ & $\begin{array}{l}\text { Casuarina papuana } \\
\text { Dacrydium } \\
\text { novoguineensis } \\
\text { A.cunninghamii }\end{array}$ \\
\hline $\begin{array}{l}\text { Disturbed dry land } \\
\text { forest }\end{array}$ & $\begin{array}{l}\text { F. microcarpa } \\
\text { Mallotus sp. } \\
\text { C. papuana }\end{array}$ & $\begin{array}{l}\text { Mallotus sp. } \\
\text { Glochidion sp. } \\
\text { C. papuana }\end{array}$ & $\begin{array}{l}\text { F. microcarpa } \\
\text { C. papuana } \\
\text { Glochidion sp. }\end{array}$ & $\begin{array}{l}\text { C. papuana } \\
\text { F. microcarpa } \\
\text { Glochidion sp. }\end{array}$ \\
\hline Pine forest & $\begin{array}{l}\text { A. cunninghamii } \\
\text { Glochidion sp } \\
\text { Tarenna confusa }\end{array}$ & $\begin{array}{l}\text { A. cunninghamii } \\
\text { Linociera montana } \\
\text { Glochidion sp. }\end{array}$ & $\begin{array}{l}\text { A. cunninghamii } \\
\text { Glochidion sp. } \\
\text { Ilex densifolia }\end{array}$ & $\begin{array}{l}\text { A. cunninghamii } \\
\text { Arthrophyllum } \\
\text { diversifolium } \\
\text { llex densifolia }\end{array}$ \\
\hline Sengon plantation & $\begin{array}{l}\text { Coffea arabica } \\
\text { Paraserianthes } \\
\text { falcataria }\end{array}$ & $\begin{array}{l}\text { C. arabica } \\
\text { P. falcataria }\end{array}$ & P. falcataria & $\begin{array}{l}\text { P. falcataria } \\
\text { C. papuana }\end{array}$ \\
\hline Complex agroforest & $\begin{array}{l}\text { C. arabica } \\
\text { P. falcataria } \\
\text { M. angulata }\end{array}$ & $\begin{array}{l}\text { C. arabica } \\
\text { M. angulata } \\
\text { P. falcataria }\end{array}$ & $\begin{array}{l}\text { C. arabica } \\
\text { Glochidion sp. } \\
\text { M. angulata }\end{array}$ & $\begin{array}{l}\text { P. falcataria } \\
\text { F. microcarpa } \\
\text { Glochidion sp. }\end{array}$ \\
\hline \multicolumn{5}{|c|}{ Merauke } \\
\hline $\begin{array}{l}\text { Undisturbed dry land } \\
\text { forest }\end{array}$ & $\begin{array}{l}\text { Dipterocarpus cornutus } \\
\text { Tabernaemontana } \\
\text { pubescens } \\
\text { Knema tomentella }\end{array}$ & $\begin{array}{l}\text { D. cornutus } \\
\text { T. pubescens } \\
\text { Uncaria gambir }\end{array}$ & $\begin{array}{l}\text { Eugenia sp. } \\
\text { K. tomentella } \\
\text { T. pubescens }\end{array}$ & $\begin{array}{l}\text { D. cornutus } \\
\text { Actiodaphne nitida } \\
\text { Euodia elleryana }\end{array}$ \\
\hline $\begin{array}{l}\text { Disturbed dry land } \\
\text { forest }\end{array}$ & $\begin{array}{l}\text { Acacia leptocarpa } \\
\text { Eucalyptus sp. } \\
\text { Buchanania } \\
\text { arborescens }\end{array}$ & $\begin{array}{l}\text { Eucalyptus sp } \\
\text { Eucalyptus pellita } \\
\text { Acacia mangium }\end{array}$ & $\begin{array}{l}\text { E. pellita } \\
\text { Eucalyptus sp. } \\
\text { Alstonia beatricis }\end{array}$ & $\begin{array}{l}\text { Eucalyptus sp. } \\
\text { E. pellita } \\
\text { A. mangium }\end{array}$ \\
\hline $\begin{array}{l}\text { Undisturbed swamp } \\
\text { forest }\end{array}$ & $\begin{array}{l}\text { Melaleuca leucadendra } \\
\text { Melaleuca cajuputi } \\
\text { Syzygium cauliflorum }\end{array}$ & $\begin{array}{l}\text { M. leucadendra } \\
\text { M.cajuputi } \\
\text { A. mangium }\end{array}$ & $\begin{array}{l}\text { M. leucadendra } \\
\text { E. pellita } \\
\text { M. cajuputi }\end{array}$ & $\begin{array}{l}\text { M. leucadendra } \\
\text { E. pellita } \\
\text { Eucalyptus sp. }\end{array}$ \\
\hline $\begin{array}{l}\text { Disturbed swamp } \\
\text { forest }\end{array}$ & $\begin{array}{l}\text { Ficus sp } \\
\text { M.cajuputi } \\
\text { E. pellita }\end{array}$ & $\begin{array}{l}\text { M. cajuputi } \\
\text { E. pellita } \\
\text { A. leptocarpa }\end{array}$ & $\begin{array}{l}\text { M. cajuputi } \\
\text { E. pellita } \\
\text { A. leptocarpa }\end{array}$ & $\begin{array}{l}\text { M. leucadendra } \\
\text { Acacia auriculiformis } \\
\text { E. pellita }\end{array}$ \\
\hline $\begin{array}{l}\text { Undisturbed } \\
\text { mangrove }\end{array}$ & $\begin{array}{l}\text { Rhizophora apiculata } \\
\text { Avicennia eucalyptifolia } \\
\text { Bruguiera cylindrica }\end{array}$ & $\begin{array}{l}\text { Rhizophora apiculata } \\
\text { Sonneratia alba } \\
\text { A. eucalyptifolia }\end{array}$ & $\begin{array}{l}\text { R. apiculata } \\
\text { Ceriops decandra } \\
\text { A. eucalyptifolia }\end{array}$ & $\begin{array}{l}\text { W. stilosa } \\
\text { R. apiculata } \\
\text { A. eucalyptifolia }\end{array}$ \\
\hline Disturbed mangrove & $\begin{array}{l}\text { B. cylindrica } \\
\text { A. eucalyptifolia } \\
\text { Hibiscus tiliaceus }\end{array}$ & $\begin{array}{l}\text { A. eucalyptifolia } \\
\text { B. cylindrica } \\
\text { C. decandra }\end{array}$ & $\begin{array}{l}\text { A.eucalyptifolia } \\
\text { B.cylindica } \\
\text { Xylocarpus } \\
\text { moluccensis }\end{array}$ & $\begin{array}{l}\text { A. eucalyptifolia } \\
\text { A. officinalis } \\
\text { B. cylindica }\end{array}$ \\
\hline Shrub on dry land & $\begin{array}{l}\text { E. pellita } \\
\text { Melaleuca viridiflora } \\
\text { Alstonia beatricis }\end{array}$ & $\begin{array}{l}\text { E. pellita } \\
\text { M. viridiflora } \\
\text { A. mangium }\end{array}$ & $\begin{array}{l}\text { E. pellita } \\
\text { M. viridiflora } \\
\text { A. mangium }\end{array}$ & $\begin{array}{l}\text { E. pellita } \\
\text { A. mangium } \\
\text { M. cajuputi }\end{array}$ \\
\hline Shrub on swamp & $\begin{array}{l}\text { M. leucadendra } \\
\text { M. cajuputi } \\
\text { M. viridiflora }\end{array}$ & $\begin{array}{l}\text { M. leucadendra } \\
\text { M. cajuputi } \\
\text { E. pellita }\end{array}$ & $\begin{array}{l}\text { M. cajuputi } \\
\text { M. leucadendra } \\
\text { M. leucadendron }\end{array}$ & $\begin{array}{l}\text { M. leucadendra } \\
\text { M. cajuputi } \\
\text { A.auriculiformis }\end{array}$ \\
\hline Complex agroforest & $\begin{array}{l}\text { N. lappaceum } \\
\text { Syzigium aqueum } \\
\text { Codiaeum variegatum }\end{array}$ & $\begin{array}{l}\text { Aleurites moluccana } \\
\text { M. sago } \\
\text { Anacardium occidentale }\end{array}$ & $\begin{array}{l}\text { Citrus aurantium } \\
\text { Psidium guajava } \\
\text { Mangifera sp. }\end{array}$ & $\begin{array}{l}\text { C. nucifera } \\
\text { Mangifera indica } \\
\text { N. lappaceum }\end{array}$ \\
\hline $\begin{array}{l}\text { Monoculture } \\
\text { treebased }\end{array}$ & $\begin{array}{l}\text { Ficus septica } \\
\text { Hevea brassiliensis } \\
\text { N. lappaceum }\end{array}$ & F. septica & $\begin{array}{l}\text { N. lappaceum } \\
\text { A. occidentale } \\
\text { H. brassiliensis }\end{array}$ & $\begin{array}{l}\text { A. occidentale } \\
\text { N. lappaceum } \\
\text { H. brassiliensis }\end{array}$ \\
\hline
\end{tabular}




\subsubsection{Species similarity among land cover types}

The species similarity among the land cover systems of Jayapura are low, except for the tree stage of cacao and complex agroforest that had the highest similarity, with a Bray-Curtis Index of 0.64 (Table 4). However, for both cacao and complex agroforest, there were no overlap species in the seedling and sapling stages. Domesticated fruits species, such as matoa (Pometia pinnata), rambutan (Nephelium lappaceum), mango (Mangifera indica) and betel nut (Areca catechu), were found in both complex and cacao agroforest.

Generally, species similarity among land cover systems (both natural and man-made ecosystems) in Jayawijaya was relatively low. Species similarity between undisturbed and disturbed forest in Jayawijaya was at the medium level for all growth stages. Similar results is occur between complex agroforest and sengon plantation for the seedling, sapling and tree stages (Table 5). No overlap species were found between sengon plantation and pine forest at the seedling, pole and tree stages.

Table 4. Matrix of species similarity among land cover systems and growth stages in Jayapura

\begin{tabular}{|c|c|c|c|c|c|}
\hline \multirow{2}{*}{$\begin{array}{l}\text { Growth } \\
\text { stage }\end{array}$} & \multirow[t]{2}{*}{ Land cover system } & \multicolumn{4}{|c|}{ Land cover system } \\
\hline & & $\begin{array}{l}\text { Undisturbed } \\
\text { dry land forest }\end{array}$ & $\begin{array}{l}\text { Complex } \\
\text { agroforest }\end{array}$ & $\begin{array}{l}\text { Sago } \\
\text { forest }\end{array}$ & $\begin{array}{l}\text { Undisturbed dry } \\
\text { land forest }\end{array}$ \\
\hline \multirow[t]{4}{*}{ Seedling } & Undisturbed dry land forest & 1 & 0.01 & 0 & 0.03 \\
\hline & Sago forest & & 1 & 0 & 0.17 \\
\hline & Cacao agroforest & & & 1 & 0 \\
\hline & Complex agroforest & & & & 1 \\
\hline \multirow[t]{4}{*}{ Sapling } & Undisturbed dry land forest & 1 & 0 & 0 & 0.04 \\
\hline & Sago forest & & 1 & 0 & 0.07 \\
\hline & Cacao agroforest & & & 1 & 0 \\
\hline & Complex agroforest & & & & 1 \\
\hline \multirow[t]{4}{*}{ Pole } & Undisturbed dry land forest & 1 & 0.01 & 0 & 0 \\
\hline & Sago forest & & 1 & 0.09 & 0.06 \\
\hline & Cacao agroforest & & & 1 & 0.15 \\
\hline & Complex agroforest & & & & 1 \\
\hline \multirow[t]{4}{*}{ Tree } & Undisturbed dry land forest & 1 & 0.01 & 0.01 & 0.02 \\
\hline & Sago forest & & 1 & 0.07 & 0.13 \\
\hline & Cacao agroforest & & & 1 & 0.64 \\
\hline & Complex agroforest & & & & 1 \\
\hline
\end{tabular}

Table 5. Matrix of species similarity among land cover systems and growth stages in Jayawijaya

\begin{tabular}{lllcccc}
\hline $\begin{array}{c}\text { Growth } \\
\text { stage }\end{array}$ & \multicolumn{1}{c}{ Land cover systems } & \multicolumn{5}{c}{ Land cover systems } \\
\cline { 3 - 7 } & $\begin{array}{c}\text { Undisturbed } \\
\text { dry land } \\
\text { forest }\end{array}$ & $\begin{array}{c}\text { Disturbed } \\
\text { dry land } \\
\text { forest }\end{array}$ & $\begin{array}{c}\text { Pine } \\
\text { forest }\end{array}$ & $\begin{array}{c}\text { Sengon } \\
\text { plantation }\end{array}$ & $\begin{array}{c}\text { Complex } \\
\text { agroforest }\end{array}$ \\
\hline Seedling & $\begin{array}{l}\text { Undisturbed dry land } \\
\text { forest }\end{array}$ & 1 & 0.48 & 0.05 & 0.11 & 0.15 \\
& Disturbed dry land forest & & 1 & 0.05 & 0.09 & 0.14 \\
& Pine forest & & & 1 & 0 & 0.02 \\
& Sengon plantation & & & & 1 & 0.46 \\
& Complex agroforest & 1 & 0.47 & 0.04 & 0.16 & 0.14 \\
\hline Sapling & Undisturbed dry land & & 1 & 0.03 & 0.06 & 0.08 \\
& forest & & & 1 & 0.01 & 0.05 \\
& Disturbed dry land forest & & & & 1 & 0.6 \\
\hline
\end{tabular}




\begin{tabular}{|c|c|c|c|c|c|c|}
\hline \multirow{2}{*}{$\begin{array}{l}\text { Growth } \\
\text { stage }\end{array}$} & \multirow[t]{2}{*}{ Land cover systems } & \multicolumn{5}{|c|}{ Land cover systems } \\
\hline & & $\begin{array}{l}\text { Undisturbed } \\
\text { dry land } \\
\text { forest }\end{array}$ & $\begin{array}{l}\text { Disturbed } \\
\text { dry land } \\
\text { forest }\end{array}$ & $\begin{array}{l}\text { Pine } \\
\text { forest }\end{array}$ & $\begin{array}{c}\text { Sengon } \\
\text { plantation }\end{array}$ & $\begin{array}{c}\text { Complex } \\
\text { agroforest }\end{array}$ \\
\hline \multirow[t]{5}{*}{ Pole } & $\begin{array}{l}\text { Undisturbed dry land } \\
\text { forest }\end{array}$ & 1 & 0.49 & 0.1 & 0.08 & 0.03 \\
\hline & Disturbed dry land forest & & 1 & 0.05 & 0.06 & 0.02 \\
\hline & Pine forest & & & 1 & 0 & 0.03 \\
\hline & Sengon plantation & & & & 1 & 0 \\
\hline & Complex agroforest & & & & & 1 \\
\hline \multirow[t]{5}{*}{ Tree } & $\begin{array}{l}\text { Undisturbed dry land } \\
\text { forest }\end{array}$ & 1 & 0.51 & 0.16 & 0.01 & 0.12 \\
\hline & Disturbed dry land forest & & 1 & 0.12 & 0.02 & 0.13 \\
\hline & Pine forest & & & 1 & 0 & 0 \\
\hline & Sengon plantation & & & & 1 & 0.45 \\
\hline & Complex agroforest & & & & & 1 \\
\hline
\end{tabular}

Mostly, species similarity among land use systems in Merauke was low for all growth stages with Bray-Curtis Similarity Index values below 0.20 (Table 6). There was medium similarity between undisturbed and disturbed mangrove for all growth stages. Shrub on swamp and disturbed dry land forest to undisturbed and disturbed swamp forest had medium to high species similarity for the sapling, pole and tree stages, but the index tended to be low for the seedling stage.

Table 6. Matrix of species similarity among land cover systems and growth stages in Merauke

\begin{tabular}{|c|c|c|c|c|c|c|c|c|c|c|c|}
\hline \multirow{2}{*}{$\begin{array}{l}\text { Growth } \\
\text { stage }\end{array}$} & \multirow[t]{2}{*}{ Land cover system } & \multicolumn{10}{|c|}{ Land cover system } \\
\hline & & UDLF & DDLF & USF & DSF & UM & DM & SDL & SSW & CAF & $\overline{T B M}$ \\
\hline \multirow[t]{10}{*}{ Seedling } & $\begin{array}{l}\text { Undisturbed dry land forest } \\
\text { (UDLF) }\end{array}$ & 1 & 0.2 & 0.06 & 0.04 & 0 & 0 & 0.08 & 0.03 & 0.01 & 0 \\
\hline & $\begin{array}{l}\text { Disturbed dry land forest } \\
\text { (DDLF) }\end{array}$ & & 1 & 0.18 & 0.15 & 0 & 0 & 0.11 & 0.13 & 0.04 & 0 \\
\hline & $\begin{array}{l}\text { Undisturbed swamp forest } \\
\text { (USF) }\end{array}$ & & & 1 & 0 & 0 & 0.46 & 0.14 & 0.18 & 0 & 0 \\
\hline & $\begin{array}{l}\text { Disturbed swamp forest } \\
\text { (DSF) }\end{array}$ & & & & 1 & 0 & 0 & 0.18 & 0.21 & 0.02 & 0.01 \\
\hline & $\begin{array}{l}\text { Undisturbed mangrove } \\
\text { (UM) }\end{array}$ & & & & & 1 & 0.19 & 0 & 0 & 0 & 0 \\
\hline & Disturbed mangrove (DM) & & & & & & 1 & 0 & 0 & 0 & 0 \\
\hline & Shrub on dry land (SDL & & & & & & & 1 & 0.14 & 0.01 & 0 \\
\hline & Shrub on swamp (SSW) & & & & & & & & 1 & 0.01 & 0 \\
\hline & Complex agroforest (CAF) & & & & & & & & & 1 & 0.04 \\
\hline & $\begin{array}{l}\text { Tree-based monoculture } \\
\text { (TBM) }\end{array}$ & & & & & & & & & & 1 \\
\hline \multirow[t]{7}{*}{ Sapling } & $\begin{array}{l}\text { Undisturbed dry land forest } \\
\text { (UDLF) }\end{array}$ & 1 & 0.25 & 0.05 & 0.02 & 0 & 0 & 0.09 & 0.01 & 0.03 & 0 \\
\hline & $\begin{array}{l}\text { Disturbed dry land forest } \\
\text { (DDLF) }\end{array}$ & & 1 & 0.21 & 0.24 & 0 & 0 & 0.32 & 0.13 & 0.06 & 0 \\
\hline & $\begin{array}{l}\text { Undisturbed swamp forest } \\
\text { (USF) }\end{array}$ & & & 1 & 0.27 & 0 & 0 & 0.15 & 0.52 & 0.03 & 0 \\
\hline & $\begin{array}{l}\text { Disturbed swamp forest } \\
\text { (DSF) }\end{array}$ & & & & 1 & 0 & 0 & 0.25 & 0.3 & 0.05 & 0 \\
\hline & $\begin{array}{l}\text { Undisturbed mangrove } \\
\text { (UM) }\end{array}$ & & & & & 1 & 0.22 & 0 & 0 & 0 & 0 \\
\hline & Disturbed mangrove (DM) & & & & & & 1 & 0 & 0 & 0 & 0 \\
\hline & Shrub on dry land (SDL & & & & & & & 1 & 0.17 & 0.04 & 0 \\
\hline
\end{tabular}




\begin{tabular}{|c|c|c|c|c|c|c|c|c|c|c|c|}
\hline \multirow{2}{*}{$\begin{array}{l}\text { Growth } \\
\text { stage }\end{array}$} & \multirow[t]{2}{*}{ Land cover system } & \multicolumn{10}{|c|}{ Land cover system } \\
\hline & & UDLF & DDLF & USF & DSF & UM & DM & SDL & SSW & CAF & TBM \\
\hline & Shrub on swamp (SSW) & & & & & & & & 1 & 0.12 & 0 \\
\hline & Complex agroforest (CAF) & & & & & & & & & 1 & 0 \\
\hline & $\begin{array}{l}\text { Tree-based monoculture } \\
\text { (TBM) }\end{array}$ & & & & & & & & & & 1 \\
\hline \multirow[t]{10}{*}{ Pole } & $\begin{array}{l}\text { Undisturbed dry land forest } \\
\text { (UDLF) }\end{array}$ & 1 & 0.22 & 0.08 & 0.06 & 0 & 0 & 0.13 & 0.04 & 0 & 0 \\
\hline & $\begin{array}{l}\text { Disturbed dry land forest } \\
\text { (DDLF) }\end{array}$ & & 1 & 0.31 & 0.3 & 0 & 0 & 0.46 & 0.2 & 0 & 0.01 \\
\hline & $\begin{array}{l}\text { Undisturbed swamp forest } \\
\text { (USF) }\end{array}$ & & & 1 & 0.38 & 0 & 0 & 0.3 & 0.37 & 0.02 & 0 \\
\hline & $\begin{array}{l}\text { Disturbed swamp forest } \\
\text { (DSF) }\end{array}$ & & & & 1 & 0 & 0 & 0.28 & 0.35 & 0.01 & 0 \\
\hline & $\begin{array}{l}\text { Undisturbed mangrove } \\
\text { (UM) }\end{array}$ & & & & & 1 & 0.38 & 0 & 0 & 0 & 0 \\
\hline & Disturbed mangrove (DM) & & & & & & 1 & 0 & 0 & 0 & 0 \\
\hline & Shrub on dry land (SDL & & & & & & & 1 & 0.23 & 0.01 & 0 \\
\hline & Shrub on swamp (SSW) & & & & & & & & 1 & 0.01 & 0 \\
\hline & Complex agroforest (CAF) & & & & & & & & & 1 & 0.07 \\
\hline & $\begin{array}{l}\text { Tree-based monoculture } \\
\text { (TBM) }\end{array}$ & & & & & & & & & & 1 \\
\hline \multirow[t]{10}{*}{ Tree } & $\begin{array}{l}\text { Undisturbed dry land forest } \\
\text { (UDLF) }\end{array}$ & 1 & 0.23 & 0.11 & 0.1 & 0 & 0 & 0.18 & 0.04 & 0 & 0 \\
\hline & $\begin{array}{l}\text { Disturbed dry land forest } \\
\text { (DDLF) }\end{array}$ & & 1 & 0.37 & 0.25 & 0 & 0 & 0.38 & 0.13 & 0.03 & 0.01 \\
\hline & $\begin{array}{l}\text { Undisturbed swamp forest } \\
\text { (USF) }\end{array}$ & & & 1 & 0.43 & 0 & 0 & 0.41 & 0.3 & 0.06 & 0 \\
\hline & $\begin{array}{l}\text { Disturbed swamp forest } \\
\text { (DSF) }\end{array}$ & & & & 1 & 0 & 0 & 0.26 & 0.6 & 0.03 & 0 \\
\hline & $\begin{array}{l}\text { Undisturbed mangrove } \\
\text { (UM) }\end{array}$ & & & & & 1 & 0.48 & 0 & 0.01 & 0.01 & 0 \\
\hline & Disturbed mangrove (DM) & & & & & & 1 & 0 & 0 & 0 & 0 \\
\hline & Shrub on dry land (SDL & & & & & & & 1 & 0.21 & 0 & 0 \\
\hline & Shrub on swamp (SSW) & & & & & & & & 1 & 0.05 & 0.01 \\
\hline & Complex agroforest (CAF) & & & & & & & & & 1 & 0.06 \\
\hline & $\begin{array}{l}\text { Tree-based monoculture } \\
\text { (TBM) }\end{array}$ & & & & & & & & & & 1 \\
\hline
\end{tabular}

\subsubsection{Species similarity among growth stages}

Species similarity among growth stages for certain land cover types varied depending on the management and human intervention during species regeneration (Table 7).

Table 7. Species similarity among growth stages in land cover types for Jayapura, Jayawijaya and Merauke

\begin{tabular}{|c|c|c|c|c|c|c|}
\hline \multirow{2}{*}{ District } & \multirow{2}{*}{ Land cover system } & \multirow{2}{*}{ Growth stage } & \multicolumn{4}{|c|}{ Growth stage } \\
\hline & & & Seedling & Sapling & Pole & Tree \\
\hline \multirow[t]{7}{*}{ Jayapura } & \multirow[t]{4}{*}{ Undisturbed dry land forest } & Seedling & 1 & 0.26 & 0.16 & 0.19 \\
\hline & & Sapling & & 1 & 0.32 & 0.22 \\
\hline & & Pole & & & 1 & 0.29 \\
\hline & & Tree & & & & 1 \\
\hline & \multirow[t]{3}{*}{ Sago forest } & Seedling & 1 & 0.15 & 0.04 & 0.07 \\
\hline & & Sapling & & 1 & 0.25 & 0.38 \\
\hline & & Pole & & & 1 & 0.56 \\
\hline
\end{tabular}




\begin{tabular}{|c|c|c|c|c|c|c|}
\hline \multirow{2}{*}{ District } & \multirow{2}{*}{ Land cover system } & \multirow{2}{*}{ Growth stage } & \multicolumn{4}{|c|}{ Growth stage } \\
\hline & & & Seedling & Sapling & Pole & Tree \\
\hline & & Tree & & & & 1 \\
\hline & \multirow[t]{4}{*}{ Cacao agroforest } & Seedling & 1 & 0.00 & 0.00 & 0.00 \\
\hline & & Sapling & & 1 & 0.00 & 0.00 \\
\hline & & Pole & & & 1 & 0.25 \\
\hline & & Tree & & & & 1 \\
\hline & \multirow[t]{4}{*}{ Complex agroforest } & Seedling & 1 & 0.18 & 0.06 & 0.08 \\
\hline & & Sapling & & 1 & 0.09 & 0.12 \\
\hline & & Pole & & & 1 & 0.55 \\
\hline & & Tree & & & & 1 \\
\hline \multirow[t]{20}{*}{ Jayawijaya } & \multirow[t]{4}{*}{ Undisturbed dry land forest } & Seedling & 1 & 0.46 & 0.22 & 0.26 \\
\hline & & Sapling & & 1 & 0.4 & 0.41 \\
\hline & & Pole & & & 1 & 0.32 \\
\hline & & Tree & & & & 1 \\
\hline & \multirow[t]{4}{*}{ Disturbed dry land forest } & Seedling & 1 & 0.62 & 0.35 & 0.50 \\
\hline & & Sapling & & 1 & 0.38 & 0.39 \\
\hline & & Pole & & & 1 & 0.4 \\
\hline & & Tree & & & & 1 \\
\hline & \multirow[t]{4}{*}{ Pines forest } & Seedling & 1 & 0.29 & 0.19 & 0.33 \\
\hline & & Sapling & & 1 & 0.47 & 0.08 \\
\hline & & Pole & & & 1 & 0.07 \\
\hline & & Tree & & & & \\
\hline & \multirow[t]{4}{*}{ Sengon plantation } & Seedling & 1 & 0.27 & 0.05 & 0.16 \\
\hline & & Sapling & & 1 & 0.28 & 0.52 \\
\hline & & Pole & & & 1 & 0.44 \\
\hline & & Tree & & & & 1 \\
\hline & \multirow{4}{*}{ Complex agroforest } & Seedling & 1 & 015 & 0.11 & 0.05 \\
\hline & & Sapling & & 1 & 0.8 & 0.08 \\
\hline & & Pole & & & 1 & 0.04 \\
\hline & & Tree & & & & 1 \\
\hline \multirow[t]{17}{*}{ Merauke } & \multirow[t]{4}{*}{ Undisturbed dry land forest } & Seedling & 1 & 0.44 & 0.31 & 0.32 \\
\hline & & Sapling & & 1 & 0.55 & 0.52 \\
\hline & & Pole & & & 1 & 0.71 \\
\hline & & Tree & & & & 1 \\
\hline & \multirow[t]{4}{*}{ Disturbed dry land forest } & Seedling & 1 & 0.52 & 0.46 & 0.4 \\
\hline & & Sapling & & 1 & 0.67 & 0.54 \\
\hline & & Pole & & & 1 & 0.67 \\
\hline & & Tree & & & & 1 \\
\hline & \multirow[t]{4}{*}{ Undisturbed swamp forest } & Seedling & 1 & 0.43 & 0.33 & 0.32 \\
\hline & & Sapling & & 1 & 0.49 & 0.39 \\
\hline & & Pole & & & 1 & 0.7 \\
\hline & & Tree & & & & 1 \\
\hline & \multirow[t]{4}{*}{ Disturbed swamp forest } & Seedling & 1 & 0.62 & 0.48 & 0.33 \\
\hline & & Sapling & & 1 & 0.45 & 0.3 \\
\hline & & Pole & & & 1 & 0.49 \\
\hline & & Tree & & & & 1 \\
\hline & Undisturbed mangrove & Seedling & 1 & 0.60 & 0.34 & 0.33 \\
\hline
\end{tabular}




\begin{tabular}{|c|c|c|c|c|c|c|}
\hline \multirow{2}{*}{ District } & \multirow{2}{*}{ Land cover system } & \multirow{2}{*}{ Growth stage } & \multicolumn{4}{|c|}{ Growth stage } \\
\hline & & & Seedling & Sapling & Pole & Tree \\
\hline & & Sapling & & 1 & 0.52 & 0.5 \\
\hline & & Pole & & & 1 & 0.67 \\
\hline & & Tree & & & & 1 \\
\hline & Disturbed mangrove & Seedling & 1 & 0.77 & 0.55 & 0.46 \\
\hline & & Sapling & & 1 & 0.46 & 0.39 \\
\hline & & Pole & & & 1 & 0.74 \\
\hline & & Tree & & & & 1 \\
\hline & Shrub on dry land & Seedling & 1 & 0.53 & 0.26 & 0.27 \\
\hline & & Sapling & & 1 & 0.51 & 0.42 \\
\hline & & Pole & & & 1 & 0.67 \\
\hline & & Tree & & & & 1 \\
\hline & Shrub on swamp & Seedling & 1 & 0.62 & 0.61 & 0.47 \\
\hline & & Sapling & & 1 & 0.69 & 0.64 \\
\hline & & Pole & & & 1 & 0.67 \\
\hline & & Tree & & & & 1 \\
\hline & Complex agroforest & Seedling & 1 & 0.33 & 0.18 & 0.21 \\
\hline & & Sapling & & 1 & 0.14 & 0.19 \\
\hline & & Pole & & & 1 & 0.27 \\
\hline & & Tree & & & & 1 \\
\hline & Tree-based monoculture & Seedling & 1 & 0.35 & 0.03 & 0.02 \\
\hline & & Sapling & & 1 & 0.00 & 0.00 \\
\hline & & Pole & & & 1 & 0.49 \\
\hline & & Tree & & & & 1 \\
\hline
\end{tabular}

Species similarity among growth stages in natural ecosystems are tend to higher than in man-made ecosystems. High similarity in natural ecosystems mostly occurred between seedlings to saplings and poles to trees. Similarity for those categories in Merauke was high, reaching above $60 \%$. However, species similarity values between seedlings to poles, seedlings to trees, saplings to poles and saplings to trees were within the range 26-69\% in the natural ecosystems of Merauke and within the range 16$32 \%$ in Jayapura, whereas in Jayawijaya the similarity between saplings to poles was around $40 \%$.

In the man-made ecosystems, the result show different trend among the districts. In complex agroforestry in Jayapura, the highest similarity occurred between the pole and tree stages followed by seedlings and sapling stages. The trend was similar for the monoculture tree-based land cover system in Merauke. On the contrary, in complex agroforest in Merauke and Jayawijaya, the highest similarity is occurred between seedlings to saplings. Poles and trees are the only growth stage that has similarity species in the cacao agroforest of Jayapura. In the monoculture tree-based system of Merauke, there was no similar of species between saplings to poles and trees.

\subsubsection{Tree diversity index}

The diversity index shows the degree of diversity in each land cover. Based on the results, natural land cover had a diversity index ranging from 1.46 to 3.92, depending on the forest type and the severity of disturbance. Man-made land cover had a wider range, with diversity index values ranging between 0.11 and 3.19. The highest diversity index was found in undisturbed dry land forest at the 
sapling level, while the lowest were found in sengon monoculture in Jayapura for the pole stage and in monoculture fruit tree-based for the sapling stage (Table 8).

Table 8. Shannon-Wiener diversity in each land covers systems and each growth stage

\begin{tabular}{|c|c|c|c|c|c|c|}
\hline District & Land cover & No. of plots & Seedling & Sapling & Pole & Tree \\
\hline \multirow[t]{4}{*}{ Jayapura } & Undisturbed dry land forest & 5 & 3.11 & 3.92 & 3.51 & 3.82 \\
\hline & Sago forest & 3 & 2.02 & 1.69 & 0.48 & 0.61 \\
\hline & Cacao agroforest & 2 & - & - & 0.85 & 1.76 \\
\hline & Complex agroforest & 6 & 2.93 & 2.91 & 0.73 & 2.09 \\
\hline \multirow[t]{5}{*}{ Jayawijaya } & Undisturbed dry land forest & 6 & 2.20 & 2.87 & 2.02 & 2.68 \\
\hline & Disturbed dry land forest & 11 & 2.52 & 2.63 & 2.54 & 2.49 \\
\hline & Pine forest & 1 & 0.64 & 1.63 & 1.47 & 0.11 \\
\hline & Sengon plantation & 1 & 0.68 & 0.67 & 0 & 0.17 \\
\hline & Complex agroforest & 2 & 0.35 & 0.94 & 0.49 & 1.47 \\
\hline \multirow[t]{10}{*}{ Merauke } & Undisturbed dry land forest & 4 & 3.03 & 3.36 & 3.60 & 3.74 \\
\hline & Disturbed dry land forest & 4 & 3.64 & 3.33 & 3.27 & 3.30 \\
\hline & Undisturbed swamp forest & 3 & 2.33 & 2.78 & 1.76 & 2.76 \\
\hline & Disturbed swamp forest & 4 & 2.93 & 2.72 & 2.78 & 2.38 \\
\hline & Undisturbed mangrove & 3 & 2.36 & 2.37 & 1.46 & 2.40 \\
\hline & Disturbed mangrove & 3 & 1.51 & 1.94 & 1.47 & 2.21 \\
\hline & Shrub on dry land & 3 & 2.30 & 1.96 & 2.15 & 2.44 \\
\hline & Shrub on swamp & 4 & 1.76 & 2.13 & 2.10 & 2.19 \\
\hline & Complex agroforest & 3 & 2.56 & 3.19 & 2.42 & 2.52 \\
\hline & Tree-based monoculture & 3 & 1.42 & 0 & 0.68 & 1.07 \\
\hline
\end{tabular}

\subsection{Carbon stock in various land cover systems}

\subsubsection{Tree biomass carbon stock}

Aboveground carbon stock in this analysis was calculated only for tree biomass. The aboveground carbon stock varied among the land covers systems of Jayapura, Jayawijaya and Merauke. Variation in the carbon stock also occurred among plot samples (Table 9). The highest aboveground carbon stock was found in complex agroforest in Jayawijaya followed by pine forest consisting of Casuarina papuana and Araucaria cunninghammii. The highest variation among plot samples occurred in disturbed dry land forest in Jayawijaya, reaching almost $80 \%$ for the average of 9 plot samples. Undisturbed dry land forest in the three districts of Papua Province contained similar aboveground carbon stock, in the range 120-135 $\mathrm{Mg} \mathrm{ha}^{-1}$. 
Table 9. Plot level aboveground carbon stock in Jayapura, Jayawijaya and Merauke

\begin{tabular}{|c|c|c|c|c|}
\hline District & Land cover & Number of plots & Carbon stock (Mg ha-1) & Standard deviation \\
\hline \multirow[t]{4}{*}{ Jayapura } & Undisturbed dry land forest & 5 & 134.5 & 53.3 \\
\hline & Sago forest & 6 & 28.2 & 26.1 \\
\hline & Cacao agroforest & 2 & 62.4 & 29.7 \\
\hline & Complex agroforest & 5 & 50.5 & 29.9 \\
\hline \multirow[t]{5}{*}{ Jayawijaya } & Undisturbed dry land forest & 6 & 131.7 & 39.7 \\
\hline & Disturbed dry land forest & 9 & 108.9 & 79.2 \\
\hline & Pines forest & 1 & 177.0 & - \\
\hline & Sengon plantation & 1 & 57.7 & - \\
\hline & Complex agroforest & 2 & 207.3 & 36.2 \\
\hline \multirow[t]{10}{*}{ Merauke } & Undisturbed dry land forest & 4 & 121.7 & 25.6 \\
\hline & Disturbed dry land forest & 4 & 80.3 & 30.0 \\
\hline & Undisturbed swamp forest & 3 & 61.7 & 15.5 \\
\hline & Disturbed swamp forest & 4 & 64.1 & 21.0 \\
\hline & Undisturbed mangrove & 3 & 100.2 & 58.5 \\
\hline & Disturbed mangrove & 3 & 74.4 & 29.9 \\
\hline & Shrub on dry land & 3 & 70.0 & 5.9 \\
\hline & Shrub on swamp & 4 & 75.9 & 27.1 \\
\hline & Complex agroforest & 3 & 26.9 & 9.7 \\
\hline & Tree-based monoculture & 3 & 44.5 & 27.6 \\
\hline
\end{tabular}

\subsubsection{Tree biomass composition}

Tree biomass carbon stock in certain land cover types was affected by the wood density, tree density and tree diameter. Average values of the wood density and tree density varied among land cover systems (Table 10).

The average wood density in the natural ecosystems in Jayapura, Jayawijaya and Merauke could be categorized from very low to medium, except for mangrove. Trees 10-30 cm DBH were dominant in almost all land cover types, except in sago forest where the DBH of trees was higher. In Jayapura and Jayawijaya, $30 \%$ of the tree biomass carbon stock was generated from trees 5-30 cm in DBH and $70 \%$ from trees $>30 \mathrm{~cm}$ DBH, but in Merauke the contribution of 5-30 $\mathrm{cm}$ and $>30 \mathrm{~cm} \mathrm{DBH}$ was $60 \%$ and $40 \%$, respectively, except for the monoculture tree-based system where $95 \%$ was generated from trees with 5-30 cm DBH. This last result clearly indicated that the monoculture tree-based systems in Merauke (rubber, cashew and rambutan) had been developed in the last two decades. 
Table 10. Average wood density and tree density by diameter class in various land cover systems in Jayapura, Jayawijaya and Merauke

\begin{tabular}{|c|c|c|c|c|c|c|c|c|}
\hline \multirow[t]{2}{*}{ District } & \multirow[t]{2}{*}{ Land cover } & \multirow{2}{*}{$\begin{array}{c}\text { Average } \\
\text { Wood } \\
\text { density } \\
\left(\mathrm{g} \mathrm{cm}^{-1}\right)\end{array}$} & \multicolumn{3}{|c|}{$\begin{array}{c}\text { Tree density per unit } \\
\text { sample }\end{array}$} & \multicolumn{2}{|c|}{$\begin{array}{l}\text { Carbon stock } \\
\left(\mathrm{Mg} \mathrm{ha}^{-1}\right)\end{array}$} & \multirow{2}{*}{$\begin{array}{c}\text { Total } \\
\text { Carbon } \\
\left(\mathrm{Mg} \mathrm{ha}^{-1}\right)\end{array}$} \\
\hline & & & $\begin{array}{l}5-10 \\
\mathrm{~cm}\end{array}$ & $\begin{array}{c}10-30 \\
\mathrm{~cm}\end{array}$ & $>30 \mathrm{~cm}$ & $\begin{array}{c}5-30 \\
\mathrm{~cm}\end{array}$ & $>30 \mathrm{~cm}$ & \\
\hline \multirow[t]{4}{*}{ Jayapura } & $\begin{array}{l}\text { Undisturbed dry land } \\
\text { forest }\end{array}$ & 0.58 & 22 & 54 & 20 & 23.4 & 111.2 & 134.6 \\
\hline & Sago forest & 0.26 & 19 & 5.3 & 9 & 12.4 & 15.9 & 28.2 \\
\hline & Cacao agroforest & 0.53 & 20 & 129 & 10 & 19.5 & 42.9 & 62.4 \\
\hline & Complex agroforest & 0.46 & 22 & 30 & 8 & 11.3 & 39.2 & 50.5 \\
\hline \multirow[t]{5}{*}{ Jayawijaya } & $\begin{array}{l}\text { Undisturbed dry land } \\
\text { forest }\end{array}$ & 0.62 & 25 & 83 & 20 & 33.1 & 98.5 & 131.7 \\
\hline & Disturbed dry land forest & 0.63 & 25 & 60 & 14 & 25.9 & 83 & 108.9 \\
\hline & Pines forest & 0.47 & 22 & 287 & 26 & 103.7 & 73.3 & 177 \\
\hline & Sengon plantation & 0.42 & 41 & 106 & 36 & 13.7 & 44 & 57.7 \\
\hline & Complex agroforest & 0.53 & 60 & 73 & 42 & 30.71 & 176.5 & 207.2 \\
\hline \multirow[t]{10}{*}{ Merauke } & $\begin{array}{l}\text { Undisturbed dry land } \\
\text { forest }\end{array}$ & 0.65 & 105 & 120 & 12 & 67.1 & 54.6 & 121.7 \\
\hline & Disturbed dry land forest & 0.65 & 98 & 70 & 7 & 47.9 & 32.4 & 80.3 \\
\hline & $\begin{array}{l}\text { Undisturbed swamp } \\
\text { forest }\end{array}$ & 0.67 & 64 & 51 & 12 & 35.5 & 26.2 & 61.7 \\
\hline & Disturbed swamp forest & 0.7 & 98 & 70 & 7 & 34.5 & 29.5 & 64.1 \\
\hline & Undisturbed mangrove & 0.79 & 69 & 75 & 7 & 55.6 & 44.5 & 100.2 \\
\hline & Disturbed mangrove & 0.72 & 78 & 85 & 6 & 56.1 & 18.3 & 74.4 \\
\hline & Shrub on dry land & 0.66 & 69 & 88 & 7 & 42 & 28 & 70 \\
\hline & Shrub on swamp & 0.66 & 107 & 64 & 5 & 59 & 16.8 & 75.9 \\
\hline & Complex agroforest & 0.6 & 16 & 16 & 6 & 8.7 & 18.2 & 26.9 \\
\hline & Tree-based monoculture & 0.62 & 46 & 129 & 2 & 42.1 & 2.5 & 44.5 \\
\hline
\end{tabular}

\section{Discussion}

\subsection{Tree diversity}

Generally, undisturbed dry land forest in the three districts of Jayapura, Jayawijaya and Merauke contained higher tree species richness than other natural ecosystems; Jayapura had the leading tree species richness followed by Merauke and Jayawijaya. The ecological condition of the sampling area was the main factor in tree species richness differentiation. The dry land forest of Jayawijaya is located at higher elevation (above 1,500 m asl) and is much affected by lower montane forest vegetation; while in Merauke, it is much affected by lowland monsoon evergreen forest that is dominated by Acacia and Melaleuca. Species richness in lower montane forest is commonly lower than in lowland forest. In Gunung Gede, West Java at 1,600 m asl, 104 species per hectare have been reported (Kartawinata 2010), whereas in Jayawijaya, the number of species was about 50\% lower.

The higher species composition among growth stages in Jayawijaya and Merauke than in Jayapura indicates a higher species overlap, but lower total species richness. The number of overlap species among growth stages in mangrove was high (in the range 70-100\%) due to the low diversity of 
mangrove species compared to other natural ecosystems. Mangrove species richness in Merauke (18 species in the undisturbed area and 14 species in the disturbed area) was higher than in undisturbed mangrove forest of Raja Ampat, where 10 species of true mangrove were reported (Prawiroatmodjo \& Kartawinata 2014). The lower species richness in man-made ecosystems of complex agroforest in Jayawijaya compared to Jayapura and Merauke may have occurred due to the availability of domesticated commercial species that were integrated in the systems and also influenced by local community culture that favored the cultivation of sweet potato as the main food.

Betel nut (Areca catechu) was a dominant species in the complex agroforest of Jayapura for all growth stages. In addition, betel nut was one of the top-three species in sago forest and cacao agroforest. Demand for betel nut for traditional consumption is high in Jayapura and the good price for betel nut (Rp. 50.000-100.000 per small plastic bag) has resulted in the local community being interested in either keeping or planting betel nut seedlings on their land (Kobepa 2016).

Araucaria cunninghammii was one of the top-three dominant species in all growth stages in the undisturbed forest of Jayawijaya. Isolated A. cunninghammii trees indicated that the area has been the site for ceremonial events. Casuarina papuana was the dominant species in the disturbed forest for all growth stages. The existence of this anthropogenic species indicates earlier settlement. The local community grows this species for building materials, firewood and fencing of their sweet potato crops (Purwanto 2003). Ficus and Glochidion were also in the top-three dominant species, indicating that forest disturbance dates back more than 20 years (Purwanto 2003). Paraserianthes falcataria was commonly found in the complex agroforest forest; nowadays this species is used as shading for new coffee systems.

The undisturbed dry land forest of Merauke was dominated by low land forest species such as Dipterocarpus cornutus in the seedling, sapling and tree stages and by Eugenia sp. in the pole stage, though $D$. cornutus was not in the top-three species. Changes in the dominance of species in certain ecosystems can indicate disturbance has occurred. In natural ecosystems, there is no single dominant species, with often hundred species sharing an area (Leigh 2004). The diversity in dominant species in all growth stages represents the complex interactions among species in natural ecosystems. In contrast, in disturbed forest, some species tend to dominate the area.

Gambir was commonly found in Merauke beside gaharu, kayu putih (Melaleuca sp.), merbau (Instia bijuga), rahai (Acacia sp.), mahosi and lawang (Wattimena 2013). The bark of gambir is extracted by the local community as a forest product used for cosmetics purposes. Extracting bark from individual poles and trees may decrease the gambir population in these stages. The dominance of fruits and other domesticated commercial species in the man-made ecosystems (complex agroforest and monoculture tree-based) was influenced by migrant communities from the government transmigration program over the last 20 years (Fitri 2015).

Dominance is an indicator of species composition in a habitat (Lohbeck et al 2014). The dominance of a species refers to its relative importance in its habitat (Chase et al 2003), which determine the degree of influence of the species in a habitat. The dominance of a certain species provides major ecosystem services, but it doesn't mean that other species have minor roles in ecosystem services.

Man-made ecosystems that focus on commercial species for economic purposes can have a high impact and result in the ecosystem having low similarity with the species in natural ecosystems. Only 
a selected few natural species were found in man-made ecosystems. Tree biodiversity in Papua is threatened by the unwise expansion of paddy rice for food security, particularly in Jayapura and Merauke, as well as by the monoculture tree-based systems of industrial forest estates. In ecosystems, trees play important roles in providing habitat to other biodiversity, both animals and other plants, even microorganisms. On the other hand, animals such as birds and bats also play important roles in the ecosystems as pollinators and in seed dispersal and pest control. Trees in the ecosystems provide services supporting biodiversity, even for human wellbeing. The more complex the biodiversity composition in a certain area indicates a more stable ecosystem.

Similarity in species among growth stages in natural ecosystems was higher than in man-made ecosystems. This indicates that species in natural ecosystems are more sustainable. Management activities in the man-made ecosystems create gaps in the growth stages of the vegetation. People tend to keep the poles and trees of certain species, but plant seedlings of different species to enrich the land for economic purposes. Non-economic species are sometimes removed during weeding. Natural species actually can survive in man-made ecosystems, as long as their seed dispersal agents still exist. The probability of a species surviving increases when the ecosystems directly adjacent are natural. Low variation in the diversity index among growth stages was found in natural land cover due to the high number of species and low evenness, as there is no single species dominant and the regeneration process is continuous. With man-made land cover, the diversity index in the growth stage was relatively high. Dominance of certain species in a certain stage occurred due to planting activity with a similar age of seedlings.

\subsection{Tree biomass carbon stock}

Tree biomass carbon stock in the undisturbed dry land forests of Jayapura, Jayawijaya and Merauke was relatively low (about $130 \mathrm{Mg} \mathrm{ha}^{-1}$ ) compared to Sumatra and Kalimantan where values of more than $200 \mathrm{Mg} \mathrm{ha}^{-1}$ have been reported (van Noordwijk et al 2002; Rahayu et al 2005). Normally Indonesian forests have been estimated to contain carbon stocks ranging from 161 to $300 \mathrm{Mg} \mathrm{ha}^{-1}$ (Murdiyarso et al 1995), but according to Lasco (2002) the carbon stock of Southeast Asian forests varied between 40 and $250 \mathrm{Mg} \mathrm{ha}^{-1}$. The average wood density of species was relatively low at $0.58 \mathrm{~g}$ $\mathrm{cm}^{-3}$ (Jayapura), $0.62 \mathrm{~g} \mathrm{~cm}^{-3}$ (Jayawijaya) and $0.65 \mathrm{~g} \mathrm{~cm}^{-3}$ (Merauke) compared to low land forest in Kalimantan (about $0.72 \mathrm{~g} \mathrm{~cm}^{-3}$ ) and this results in a lower total carbon stock (Rahayu et al 2005; Rahayu et al 2016).

Both the undisturbed and disturbed swamp forest of Merauke contained low carbon stock (about 60 $\mathrm{Mg} \mathrm{ha}^{-1}$ ) compared to a previous survey, where $200 \mathrm{Mg} \mathrm{ha}^{-1}$ in undisturbed swamp and $92 \mathrm{Mg} \mathrm{ha}^{-1}$ in disturbed swamp were reported (Rahayu \& Harja 2012). The tree density in each plot may have affected the total tree biomass and hence the carbon stock. However, more samples plots are needed to represent the large area of swamp forest in Merauke. Additional plot samples will affect the average carbon stock estimates in certain areas due to plot variation in tree density and species composition. However, the shrub on swamp area was $100 \%$ higher than that in a previous study (Rahayu \& Harja 2012), due to the different age of shrubs regenerating in the plots and misclassification of either disturbed swamp forest or shrub on swamp.

In this study, the tree biomass carbon stock in the undisturbed mangrove of Merauke was similar to a previous survey with values in the range $100-120 \mathrm{Mg} \mathrm{ha}^{-1}$, but was $100 \%$ higher for disturbed 
mangrove (Rahayu \& Harja 2012). The distribution of plot samples in various disturbance levels affected the estimation of tree biomass carbon stock, since the level of disturbance influences the tree density. variation in the tree biomass carbon stock in the complex agroforest system of Jayapura, Jayawijaya and Merauke was influenced by the species composition in the systems. Cacao and other fruits were dominant species in Jayapura, in contrast to mixed fruits in Merauke and coffee with sengon as a shading tree in Jayawijaya. The high population of large sengon trees ( $>30 \mathrm{~cm} \mathrm{DBH})$ contributed to the high carbon stock. The monoculture tree-based system in Merauke was categorized as having low carbon stock. The availability of established old systems for sampling in Merauke was a constraint. Additional plot samples covering the full range for young to old plantation is needed for further analysis. The carbon stock composition based on tree size indicates the regeneration period. If the contribution to tree biomass carbon stock in bigger trees (> $30 \mathrm{~cm} \mathrm{DBH})$ is higher, then the system has been established for a long time. In contrast, if there are many smaller trees (5-30 cm DBH), then the system is relatively young.

\section{Conclusions}

Tree species richness in the natural ecosystems of Jayapura was higher than in Jayawijaya and Merauke due to biophysical conditions. The most important species in the man-mad ecosystems varied depending on the market conditions, with betel nut predominating in Jayapura while the most important was coffee in Jayawijaya and mixed fruits in Merauke. Natural ecosystems provide the best area for tree species conservation, in contrast to man-made ecosystems. Undisturbed dry land forest in Jayapura and Merauke contained the highest carbon stock compared to the other ecosystems, but pines forest and complex agroforest contained the highest carbon stock in Jayawijaya.

\section{Suggestion}

For further analysis, additional sampling plots are needed to reduce bias.

\section{References}

Chave J, Andalo C, Brown S, Cairns MA, Chambers JQ, et al. 2005. Tree allometry and improved estimation of carbon stocks and balance in tropical forests. Oecologia 145:87-99. DOI: 10.1007/s00442-005-0100-x.

Chase JM, Leibold MA. 2003. Ecological Niches: Linking Classical and Contemporary Approaches. Chicagro: University of Chicago Press http://dx.doi.org/10.7208/chicago/9780226101811.001.0001

Cowell RK. 2009. Biodiversity: Concepts, Patterns and Measurement. In: Levin SA (ed.). The Princeton Guide to Ecology. Princeton: Princeton University Press. pp. 257-263.

Curtis JT, McIntosh RP. 1950. The interrelations of certain analytic and synthetic phytosociological characters. Ecology, 31: 434-455. 
Fitri L. 2015. Nostalgia Kampung Jawa di Merauke. Kompasiana 26 Juni 2015. http://www.kompasiana.com/listiyofitri/nostalgia-kampung-jawadimerauke_5500dc42a33311351951013d

Hairiah K, Rahayu S. 2007. Pengukuran 'karbon tersimpan' di berbagai macam penggunaan lahan. Bogor, Indonesia: World Agroforestry Centre -ICRAF, SEA Regional Office, University of Brawijaya, Unibraw. 77 pp.

Hairiah K, Dewi S, Agus F, velarde S, Ekadinata A, Rahayu S, et al. 2011. Measuring Carbon Stocks Across Land Use Systems: A Manual. Bogor, Indonesia: World Agroforestry Centre (ICRAF), SEA Regional Office, 154 pp.

Harris NL, Brown S, Hagen SC, Saatchi SS, Petrova S, et al. 2012. Baseline map of carbon emissions from deforestation in tropical regions. Science 336: 1573-1576.

Kartawinata K. 2010. Dua Abad mengungkap Kekayaan Flora dan Ekosistem di Indonesia. Sarwono Prawirohardjo Memorial Lecture X, Jakarta, Indonesia: Lembaga Ilmu Pengetahuan Indonesia.

Kobepa F. 2016. Buah Pinnang Menyatu dengan Kehidupan Orang Papua. Media Informasi Online, Papualives.com, 6 March 2016.

Lasco RD. 2002. Forest carbon budgets in Southeast Asia following harvesting and land cover change. In: Impacts of land use change on the terrestrial carbon cycle in the Asia Pacific Region. Science in China 45: 76-86.

Leigh D, Puyravaud D, Terborgh S, Wright SJ. 2004. Why do some tropical forests have so many species of trees? Biotropica 36: 447-473.

Lohbeck M, Poorter L, Martínez-Ramos M, Rodriguez-velázquez J, van Breugel M, et al. 2014. Changing drivers of species dominance during tropical forest succession. Functional Ecology 28: 1052-1058. http://dx.doi.org/10.1111/1365-2435.12240

Murdiyarso D, Wasrin UR. 1995. Estimating land use change and carbon release from tropical forest conversion using remote sensing technique. Journal of Biogeography 22: 715-721.

Prawiroatmodjo S, Kartawinata K. 2014. Floristic diversity and structural characteristics of mangrove forest of Raja Ampat, West Papua, Indonesia. Reinwardtia 14(1): 170-180.

Purwanto Y. 2003. Studi etnoekologi masyarakat Dani-Baliem dan perubahan lingkungan di Lembah Baliem, Jayawijaya, Irian Jaya. Berita Biologi 6(5): 661-678.

Rahayu S, Lusiana B and van Noordwijk M. 2005. Carbon Stock Monitoring in Nunukan, East Kalimantan: A Spatial and Modelling Approach. World Agroforestry Centre, SE Asia, Bogor, Indonesia: 21-34.

Rahayu S, Harja D. 2012. A Study of Carbon Stock Appraisal: Average Carbon Stock of various land Cover in Merauke, Papua Province. World Agroforestry Centre (ICRAF) - The World Wildlife Fund (WWF). Technical Report.

Rahayu S, Pambudi, S, van Noordwijk M, Harja D. 2016. Wood density as a proxy for tree functional group recovery after forest disturbance in lowland forest of East Kalimantan, Indonesia. Paper presented at The $6^{\text {th }}$ International Conference on Mathematics and Natural Sciences 2016 (ICMNS 2016), 02-03 November 2016, Bandung, Indonesia.

Wattimena MC. 2013. Perspektif Kearifan Tempat Penting Suku Malind sebagai Arahan dalam Penyusunan Rencana Detil Tata Ruang Wilayah Kabupaten Merauke. Bogor, Indonesia: Institut Pertanian Bogor. Thesis

van Noordwijk M, Rahayu S, Hairiah K, Wulan YC, Farida A, et al. 2002. Carbon stock assessment for a forestto-coffee conversion landscape in Sumberjaya (Lampung, Indonesia): from allometric equation to land use change analysis. Science in China 45: 75-86. 
Table Appendix 1. Tree species in each land cover type in Jayapura District

\begin{tabular}{|c|c|c|c|c|c|}
\hline \multirow[t]{2}{*}{ No. } & \multirow[t]{2}{*}{ Tree Species } & \multicolumn{4}{|c|}{ Land cover } \\
\hline & & $\begin{array}{l}\text { Undisturbed } \\
\text { dry land } \\
\text { forest }\end{array}$ & Sagoo & $\begin{array}{c}\text { Cacao } \\
\text { agroforest }\end{array}$ & $\begin{array}{c}\text { Complex } \\
\text { agroforest }\end{array}$ \\
\hline 1 & Actinodaphne akoensis & v & & & \\
\hline 2 & Adenanthera sp. & v & & & \\
\hline 3 & Agathis borneensis & $\mathrm{v}$ & & & \\
\hline 4 & Agathis sp. & v & & & \\
\hline 5 & Agathis sp.1 & $\mathrm{v}$ & & & \\
\hline 6 & Aglaia sp.1 & v & v & & \\
\hline 7 & Aglaia sp.2 & $\mathrm{v}$ & & & \\
\hline 8 & Aglaia sp.3 & v & & & \\
\hline 9 & Aglaia sp.4 & $\mathrm{v}$ & & & \\
\hline 10 & Agrostistachys sp. & $v$ & & & \\
\hline 11 & Alphitonia macrocarpa & & & v & \\
\hline 12 & Alseodaphne laevis & v & & & \\
\hline 13 & Alseodaphne umbelliflora & v & & & \\
\hline 14 & Alstonia sp. & & v & & \\
\hline 15 & Anisoptera thurifera & v & & & \\
\hline 16 & Annona muricata & & & v & v \\
\hline 17 & Anthocephalus chinensis & v & & & \\
\hline 18 & Aporosa sp.1 & v & v & & v \\
\hline 19 & Aporosa sp.2 & v & & & \\
\hline 20 & Areca catechu & & v & v & v \\
\hline 21 & Arecaceae & v & & & \\
\hline 22 & Artocarpus altilis & v & v & & v \\
\hline 23 & Artocarpus sp. & & $v$ & v & v \\
\hline 24 & Averrhoa carambola & & & v & \\
\hline 25 & Bambusa sp. & & v & & \\
\hline 26 & Barringtonia lauterbachii & $\mathrm{v}$ & & & \\
\hline 27 & Breynia cernua & $v$ & & & \\
\hline 28 & Bruguiera sexangula & v & & & \\
\hline 29 & Burckella sp. & $\mathrm{v}$ & & & \\
\hline 30 & Calophyllum soulattri & v & & & v \\
\hline 31 & Calophyllum sp.2 & $\mathrm{v}$ & & & \\
\hline 32 & Canarium denticulatum & & & & v \\
\hline 33 & Canarium littorale & v & & & \\
\hline 34 & Canarium maluense & v & & & \\
\hline 35 & Canarium sp. & $\mathrm{v}$ & & & \\
\hline 36 & Canarium sylvestre & v & & & \\
\hline 37 & Carica papaya & & & & v \\
\hline
\end{tabular}




\begin{tabular}{|c|c|c|c|c|c|}
\hline \multirow[t]{2}{*}{ No. } & \multirow[t]{2}{*}{ Tree Species } & \multicolumn{4}{|c|}{ Land cover } \\
\hline & & $\begin{array}{c}\text { Undisturbed } \\
\text { dry land } \\
\text { forest }\end{array}$ & Sagoo & $\begin{array}{c}\text { Cacao } \\
\text { agroforest }\end{array}$ & $\begin{array}{c}\text { Complex } \\
\text { agroforest }\end{array}$ \\
\hline 38 & Casuarina junghuhniana & v & & & \\
\hline 39 & Cerbera sp.1 & v & v & & \\
\hline 40 & Champereia manillana & $\mathrm{v}$ & & & \\
\hline 41 & Chionanthus sp.1 & v & & & \\
\hline 42 & Chionanthus sp.2 & v & & & \\
\hline 43 & Cinnamomum sp. & v & & & \\
\hline 44 & Cocos nucifera & & v & v & v \\
\hline 45 & Coffea sp. & & & & $v$ \\
\hline 46 & Cryptocarya sp.1 & v & & & \\
\hline 47 & Cyathocalyx cauliflorus & v & & & \\
\hline 48 & Cynometra sp. & & & & v \\
\hline 49 & Diospyros lolin & v & & & \\
\hline 50 & Dipterocarpus validus & v & & & \\
\hline 51 & Durio sp. & & & v & v \\
\hline 52 & Dysoxylum arborescens & v & & & \\
\hline 53 & Dysoxylum sp.1 & v & & & \\
\hline 54 & Elaeocarpus cf. sepikanus & v & & & \\
\hline 55 & Elaeocarpus sp. & v & & & \\
\hline 56 & Elattostachys sp. & v & & & \\
\hline 57 & Endiandra cf. rubescens & v & & & \\
\hline 58 & Erythroxylum ecarinatum & v & & & \\
\hline 59 & Eugenia sp. & & & $\mathrm{v}$ & $\mathrm{v}$ \\
\hline 60 & Evodia sp. & v & & & \\
\hline 61 & Fagraea racemosa & v & & & \\
\hline 62 & Fagraea sp. & v & & & \\
\hline 63 & Ficus glandulifera & & & v & \\
\hline 64 & Ficus montana & & & & $v$ \\
\hline 65 & Ficus sp.1 & v & & & \\
\hline 66 & Ficus sp.2 & & & & $v$ \\
\hline 67 & Ficus sp.3 & & & & v \\
\hline 68 & Ficus sp.4 & v & & & \\
\hline 69 & Ficus tinctoria & v & & & \\
\hline 70 & Ficus vasculosa & v & & & \\
\hline 71 & Garcinia cf. riedeliana & v & & & \\
\hline 72 & Garcinia parvifolia & v & & & \\
\hline 73 & Garcinia sp. & v & & & \\
\hline 74 & Gironniera nervosa & v & & & \\
\hline 75 & Gironniera subaequalis & v & & & \\
\hline 76 & Gliricidia sepium & & & v & $v$ \\
\hline
\end{tabular}




\begin{tabular}{|c|c|c|c|c|c|}
\hline \multirow[t]{2}{*}{ No. } & \multirow[t]{2}{*}{ Tree Species } & \multicolumn{4}{|c|}{ Land cover } \\
\hline & & $\begin{array}{l}\text { Undisturbed } \\
\text { dry land } \\
\text { forest }\end{array}$ & Sagoo & $\begin{array}{c}\text { Cacao } \\
\text { agroforest }\end{array}$ & $\begin{array}{c}\text { Complex } \\
\text { agroforest }\end{array}$ \\
\hline 77 & Glochidion cf. arborescens & $\mathrm{v}$ & & & \\
\hline 78 & Gmelina arborea & & $\mathrm{v}$ & v & \\
\hline 79 & Gnetum gnemon & $\mathrm{v}$ & & & $\mathrm{v}$ \\
\hline 80 & Gomphandra australiana & v & & & \\
\hline 81 & Gonystylus & v & & & \\
\hline 82 & Guioa membranifolia & v & & & \\
\hline 83 & Guioa pteropoda & $\mathrm{v}$ & & & \\
\hline 84 & Gymnacranthera paniculata & v & & & \\
\hline 85 & Haplolobus cf. floribundus & $\mathrm{v}$ & & & \\
\hline 86 & Haplolobus floribundus & v & & & \\
\hline 87 & $\begin{array}{l}\text { Haplolobus floribundus ssp. } \\
\text { floribundus }\end{array}$ & v & & & \\
\hline 88 & Haplolobus pachypodus & v & & & \\
\hline 89 & Haplolobus sp. & v & & & \\
\hline 90 & Haplolobus sp.1 & v & & & \\
\hline 91 & Haplolobus sp.2 & $\mathrm{v}$ & & & \\
\hline 92 & Hibiscus sp. & & & & v \\
\hline 93 & Homalium foetidum & & $\mathrm{v}$ & & \\
\hline 94 & Hopea sp.1 & v & & & \\
\hline 95 & Horsfieldia lancifolia & $\mathrm{v}$ & & & \\
\hline 96 & Inocarpus fagiferus & $\mathrm{v}$ & $\mathrm{v}$ & & v \\
\hline 97 & loides sp. & $\mathrm{v}$ & & & \\
\hline 98 & Ixora cf. amboinica & $\mathrm{v}$ & & & \\
\hline 99 & Ixora sp. & $\mathrm{v}$ & & & \\
\hline 100 & Koilodepas sp. & $\mathrm{v}$ & & & \\
\hline 101 & Lansium domesticum & & $v$ & & $v$ \\
\hline 102 & Lasianthus sp. & v & & & \\
\hline 103 & Leucaena leucocephala & & & & $v$ \\
\hline 104 & Litsea mappacea & v & & & \\
\hline 105 & Macaranga hypoleuca & & v & & \\
\hline 106 & Macaranga inermis & v & & & \\
\hline 107 & Macaranga sp. & & & & $v$ \\
\hline 108 & Mangifera sp. & & & v & v \\
\hline 109 & Manihot sp. & & & & $v$ \\
\hline 110 & Maranthes corymbosa & v & & v & \\
\hline 111 & Metroxylon sagu & & $\mathrm{v}$ & & \\
\hline 112 & Mischocarpus sundaicus & v & & & \\
\hline 113 & Morinda citrifolia & & & & $v$ \\
\hline 114 & Musa sp. & & $v$ & & v \\
\hline
\end{tabular}




\begin{tabular}{|c|c|c|c|c|c|}
\hline \multirow[t]{2}{*}{ No. } & \multirow[t]{2}{*}{ Tree Species } & \multicolumn{4}{|c|}{ Land cover } \\
\hline & & $\begin{array}{l}\text { Undisturbed } \\
\text { dry land } \\
\text { forest }\end{array}$ & Sagoo & $\begin{array}{c}\text { Cacao } \\
\text { agroforest }\end{array}$ & $\begin{array}{c}\text { Complex } \\
\text { agroforest }\end{array}$ \\
\hline 115 & Myristica subalulata & & $v$ & & \\
\hline 116 & Nephelium lappaceum & & v & $\mathrm{v}$ & v \\
\hline 117 & Nephelium sp. & & & $\mathrm{v}$ & \\
\hline 118 & Oncosperma tigillarium & & $\mathrm{v}$ & & \\
\hline 119 & Pandanus sp. & v & & & \\
\hline 120 & Paratocarpus venenosa & $v$ & & & \\
\hline 121 & Parinari sp. & v & & & \\
\hline 122 & Parkia speciosa & & $v$ & & v \\
\hline 123 & Phyllanthus cf. niruri & & & & v \\
\hline 124 & Pimelodendron amboinicum & $v$ & & & \\
\hline 125 & Planchonella cf. firma & v & v & & \\
\hline 126 & Planchonella firma & $v$ & & & \\
\hline 127 & Planchonella sp.1 & v & & & \\
\hline 128 & Planchonia papuana & & & v & \\
\hline 129 & Plectronia horrida & v & & & \\
\hline 130 & Pleomele angustifolia & $v$ & & & \\
\hline 131 & Pometia pinnata & v & v & v & v \\
\hline 132 & Psychotria sp.1 & $v$ & & & \\
\hline 133 & Psychotria sp.2 & $v$ & & & \\
\hline 134 & Psychotria sp.1 & v & & & \\
\hline 135 & Quisqualis indica & $v$ & & & \\
\hline 136 & Rhodamnia cinerea & v & & & \\
\hline 137 & Salacia sp.1 & $v$ & & & \\
\hline 138 & Sloetia elongata & v & & & \\
\hline 139 & Smilax sp.1 & $v$ & & & \\
\hline 140 & Smilax sp. 2 & $v$ & & & \\
\hline 141 & Stemonurus monticola & $v$ & & & \\
\hline 142 & Sterculia ceramica & & $v$ & & v \\
\hline 143 & Sterculia cf. macrophylla & v & & & \\
\hline 144 & Syzygium anomalum & v & & & \\
\hline 145 & Syzygium aqueum & & & v & \\
\hline 146 & Syzygium cf. decipiens & $v$ & & & \\
\hline 147 & Syzygium fibrosum & $v$ & & & \\
\hline 148 & Syzygium malaccense & & & & v \\
\hline 149 & Syzygium naiadum & v & & & \\
\hline 150 & Syzygium sp.1 & v & & & v \\
\hline 151 & Syzygium sp.2 & $v$ & & & v \\
\hline 152 & Syzygium sp.3 & v & & & \\
\hline 153 & Syzygium sp.5 & v & & & \\
\hline
\end{tabular}




\begin{tabular}{|c|c|c|c|c|c|}
\hline \multirow[t]{2}{*}{ No. } & \multirow[t]{2}{*}{ Tree Species } & \multicolumn{4}{|c|}{ Land cover } \\
\hline & & $\begin{array}{c}\text { Undisturbed } \\
\text { dry land } \\
\text { forest }\end{array}$ & Sagoo & $\begin{array}{c}\text { Cacao } \\
\text { agroforest }\end{array}$ & $\begin{array}{c}\text { Complex } \\
\text { agroforest }\end{array}$ \\
\hline 154 & Teijsmaniodendron & $\mathrm{v}$ & & & \\
\hline 155 & Terminalia kaernbachii & v & & & \\
\hline 156 & Ternstroemia merrilliana & v & & & \\
\hline 157 & Theobroma cacao & & $v$ & $v$ & $v$ \\
\hline 158 & Unidentified_1 & v & & & \\
\hline 159 & Unidentified_10 & v & & & \\
\hline 160 & Unidentified_11 & v & & & \\
\hline 161 & Unidentified_12 & v & & & \\
\hline 162 & Unidentified_13 & v & & & \\
\hline 163 & Unidentified_14 & v & & & \\
\hline 164 & Unidentified_16 & v & & & \\
\hline 165 & Unidentified_17 & v & & & \\
\hline 166 & Unidentified_18 & v & & & \\
\hline 167 & Unidentified_19 & v & & & \\
\hline 168 & Unidentified_2 & v & & & \\
\hline 169 & Unidentified_20 & v & & & \\
\hline 170 & Unidentified_21 & v & & & \\
\hline 171 & Unidentified_22 & v & & & \\
\hline 172 & Unidentified_23 & v & & & \\
\hline 173 & Unidentified_24 & $\mathrm{v}$ & & & \\
\hline 174 & Unidentified_25 & v & & & \\
\hline 175 & Unidentified_26 & $\mathrm{v}$ & & & \\
\hline 176 & Unidentified_27 & v & & & \\
\hline 177 & Unidentified_28 & & & & $\mathrm{v}$ \\
\hline 178 & Unidentified_29 & v & & & \\
\hline 179 & Unidentified_30 & $\mathrm{v}$ & & & \\
\hline 180 & Unidentified_31 & v & & & \\
\hline 181 & Unidentified_32 & $\mathrm{v}$ & & & \\
\hline 182 & Unidentified_33 & v & & & \\
\hline 183 & Unidentified_34 & v & & & \\
\hline 184 & Unidentified_35 & & v & & \\
\hline 185 & Unidentified_36 & & & & v \\
\hline 186 & Unidentified_37 & v & & & \\
\hline 187 & Unidentified_38 & v & & & \\
\hline 188 & Unidentified_39 & & v & & \\
\hline 189 & Unidentified_4 & v & & & \\
\hline 190 & Unidentified_40 & v & & & \\
\hline 191 & Unidentified_41 & v & & & \\
\hline 192 & Unidentified_42 & v & & & \\
\hline
\end{tabular}




\begin{tabular}{|c|c|c|c|c|c|}
\hline \multirow[t]{2}{*}{ No. } & \multirow[t]{2}{*}{ Tree Species } & \multicolumn{4}{|c|}{ Land cover } \\
\hline & & $\begin{array}{c}\text { Undisturbed } \\
\text { dry land } \\
\text { forest }\end{array}$ & Sagoo & $\begin{array}{c}\text { Cacao } \\
\text { agroforest }\end{array}$ & $\begin{array}{c}\text { Complex } \\
\text { agroforest }\end{array}$ \\
\hline 193 & Unidentified_43 & $\mathrm{v}$ & & & \\
\hline 194 & Unidentified_44 & v & & & \\
\hline 195 & Unidentified_45 & $\mathrm{v}$ & & & \\
\hline 196 & Unidentified_46 & $v$ & & & \\
\hline 197 & Unidentified_47 & v & & & \\
\hline 198 & Unidentified_48 & v & & & \\
\hline 199 & Unidentified_49 & v & & & \\
\hline 200 & Unidentified_5 & v & & & \\
\hline 201 & Unidentified_50 & v & & & \\
\hline 202 & Unidentified_51 & v & & & \\
\hline 203 & Unidentified_52 & v & & & \\
\hline 204 & Unidentified_53 & v & & & \\
\hline 205 & Unidentified_54 & v & & & \\
\hline 206 & Unidentified_55 & v & & & \\
\hline 207 & Unidentified_56 & v & & & \\
\hline 208 & Unidentified_57 & v & & & \\
\hline 209 & Unidentified_58 & v & & & \\
\hline 210 & Unidentified_59 & & & & v \\
\hline 211 & Unidentified_6 & v & & & \\
\hline 212 & Unidentified_60 & $\mathrm{v}$ & & & \\
\hline 213 & Unidentified_61 & v & & & \\
\hline 214 & Unidentified_62 & v & & & \\
\hline 215 & Unidentified_63 & & & & v \\
\hline 216 & Unidentified_65 & & & & v \\
\hline 217 & Unidentified_66 & & & & $v$ \\
\hline 218 & Unidentified_67 & v & & & \\
\hline 219 & Unidentified_68 & v & & & \\
\hline 220 & Unidentified_69 & v & & & \\
\hline 221 & Unidentified_7 & v & & & \\
\hline 222 & Unidentified_70 & v & & & \\
\hline 223 & Unidentified_71 & v & & & \\
\hline 224 & Unidentified_72 & v & & & \\
\hline 225 & Unidentified_73 & v & & & \\
\hline 226 & Unidentified_74 & v & & & \\
\hline 227 & Unidentified_76 & v & & & \\
\hline 228 & Unidentified_77 & v & & & \\
\hline 229 & Unidentified_78 & v & & & \\
\hline 230 & Unidentified_79 & v & & & \\
\hline 231 & Unidentified_8 & $\mathrm{v}$ & & & \\
\hline
\end{tabular}




\begin{tabular}{|c|c|c|c|c|c|}
\hline \multirow[t]{2}{*}{ No. } & \multirow[t]{2}{*}{ Tree Species } & \multicolumn{4}{|c|}{ Land cover } \\
\hline & & $\begin{array}{l}\text { Undisturbed } \\
\text { dry land } \\
\text { forest }\end{array}$ & Sagoo & $\begin{array}{c}\text { Cacao } \\
\text { agroforest }\end{array}$ & $\begin{array}{c}\text { Complex } \\
\text { agroforest }\end{array}$ \\
\hline 232 & Unidentified_80 & & & & v \\
\hline 233 & Unidentified_81 & v & & & \\
\hline 234 & Unidentified_9 & $\mathrm{v}$ & & & \\
\hline 235 & Unidentified_a & & & $v$ & \\
\hline 236 & Unidentified_saweng & & & v & \\
\hline 237 & Unidentified_SR3 & & & $v$ & \\
\hline 238 & Villebrunea rubescens & & & & v \\
\hline \multirow[t]{2}{*}{239} & Voacanga grandifolia & & & & $v$ \\
\hline & Number of species & 183 & 26 & 22 & 45 \\
\hline
\end{tabular}


Table Appendix 2. Tree species in each land cover type in Jayawijaya District

\begin{tabular}{|c|c|c|c|c|c|c|}
\hline \multirow[t]{2}{*}{ No } & \multirow[t]{2}{*}{ Tree Species } & \multicolumn{5}{|c|}{ Land Cover } \\
\hline & & $\begin{array}{l}\text { Undisturbed } \\
\text { dry land } \\
\text { forest }\end{array}$ & $\begin{array}{c}\text { Disturbed } \\
\text { dry land } \\
\text { forest }\end{array}$ & $\begin{array}{l}\text { Pine } \\
\text { forest }\end{array}$ & $\begin{array}{c}\text { Sengon } \\
\text { plantation }\end{array}$ & $\begin{array}{c}\text { Complex } \\
\text { Agroforest }\end{array}$ \\
\hline 1 & Adinandra sp. & v & v & & & \\
\hline 2 & Aphanomyrtus sp. & v & & & & \\
\hline 3 & Araucaria cunninghamii & $v$ & v & v & & v \\
\hline 4 & Arthrophyllum diversifolium & & $v$ & $v$ & & \\
\hline 5 & Artocarpus altilis & $v$ & & & & \\
\hline 6 & Bambusa & & v & & & \\
\hline 7 & Bischofia papuana & v & $\mathrm{v}$ & & & v \\
\hline 8 & Buddleja asiatica & v & v & & & \\
\hline 9 & Callicarpa sp. & $v$ & & & & \\
\hline 10 & Casuarina papuana & v & v & & v & v \\
\hline 11 & Chionanthus sp. & v & v & v & & \\
\hline 12 & Coffea arabica & v & v & & v & v \\
\hline 13 & Coffea sp. & v & v & & & \\
\hline 14 & Commersonia sp. & v & & & & \\
\hline 15 & Cynometra sp. & & v & & & \\
\hline 16 & Cyphomandra betacea & & & & & v \\
\hline 17 & Cyrtandra sp. & v & $\mathrm{v}$ & & & \\
\hline 18 & Dacrydium novaguineensis & v & v & & & \\
\hline 19 & Dodonaea viscosa & v & $\mathrm{v}$ & & & \\
\hline 20 & Elaeocarpus obtusa & & v & & & \\
\hline 21 & Erythrina lithosperma & & $\mathrm{v}$ & & & \\
\hline 22 & Eugenia paucipunctata & v & v & & & \\
\hline 23 & Fagraea elliptica & v & $\mathrm{v}$ & & & \\
\hline 24 & Fagraea sp. & & v & & & \\
\hline 25 & Ficus glomerata & v & & & & \\
\hline 26 & Ficus microcarpa & v & v & & & v \\
\hline 27 & Ficus quercifolia & v & $\mathrm{v}$ & & & \\
\hline 28 & Firmania sp. & v & & & & \\
\hline 29 & Glochidion sp. & v & $\mathrm{v}$ & v & & v \\
\hline 30 & Glochidion sp.1 & v & v & & & v \\
\hline 31 & Glochidion sp.2 & v & v & & & v \\
\hline 32 & Glochidion sp.3 & v & v & v & & \\
\hline 33 & Grevillea papuana & & v & & & \\
\hline 34 & Haeckeria sp. & & v & & & \\
\hline 35 & Heckeria peltata & & v & & & \\
\hline 36 & Heckeria sp. & v & & & & \\
\hline 37 & Homalanthus sp & & $v$ & & & \\
\hline
\end{tabular}




\begin{tabular}{|c|c|c|c|c|c|c|}
\hline \multirow[t]{2}{*}{ No } & \multirow[t]{2}{*}{ Tree Species } & \multicolumn{5}{|c|}{ Land Cover } \\
\hline & & $\begin{array}{l}\text { Undisturbed } \\
\text { dry land } \\
\text { forest }\end{array}$ & $\begin{array}{c}\text { Disturbed } \\
\text { dry land } \\
\text { forest } \\
\end{array}$ & $\begin{array}{l}\text { Pine } \\
\text { forest }\end{array}$ & $\begin{array}{c}\text { Sengon } \\
\text { plantation }\end{array}$ & $\begin{array}{l}\text { Complex } \\
\text { Agroforest }\end{array}$ \\
\hline 38 & Ilex densifolia & & $v$ & $v$ & & \\
\hline 39 & Lansium domesticum & $v$ & $v$ & $v$ & & \\
\hline 40 & Linociera montana & $v$ & $v$ & $v$ & & $v$ \\
\hline 41 & Lithocarpus rufovillosus & $v$ & $v$ & & & \\
\hline 42 & Litsea sp. & $v$ & $v$ & & & \\
\hline 43 & Mallotus sp. & $v$ & $v$ & & & $v$ \\
\hline 44 & Melastoma malabthricum & & $v$ & & & \\
\hline 45 & Meliosma angulata & $v$ & v & & & $v$ \\
\hline 46 & Meliosma sp. & & $v$ & & & \\
\hline 47 & Musa sp. & $v$ & v & & & \\
\hline 48 & Mussaenda frondosa & $v$ & & & & \\
\hline 49 & Nothofagus recurva & v & & & & \\
\hline 50 & Paraserianthes falcataria & $v$ & $v$ & $v$ & $v$ & $v$ \\
\hline 51 & Paraserianthes minahassae & & & & & $v$ \\
\hline 52 & Payena leerii & & $v$ & & & \\
\hline 53 & Persea americana & & & & & $v$ \\
\hline 54 & Plectronia horrida & $v$ & & & & \\
\hline 55 & Saurauia sp. & $v$ & $v$ & & & \\
\hline 56 & Schefflera rigida & $v$ & $v$ & & & \\
\hline 57 & Solanum torvum & $v$ & & & & \\
\hline 58 & Sycopsis dunnii & v & & & & \\
\hline 59 & Tarenna confusa & $v$ & v & $v$ & & \\
\hline 60 & Timonius sp. & $v$ & $v$ & & & \\
\hline 61 & Trimenia papuana & & $v$ & & & \\
\hline 62 & Unidentified 82 & & $v$ & & & \\
\hline 63 & Unidentified 83 & & v & & & \\
\hline 64 & Unidentified_42 & $v$ & $v$ & & & \\
\hline 65 & Unidentified_44 & $v$ & $v$ & & & \\
\hline 66 & Unidentified_46 & $v$ & & & & \\
\hline 67 & Unidentified_48 & & & & & $v$ \\
\hline 68 & Unidentified_79 & & $v$ & & & \\
\hline 69 & Unidentified_8 & $v$ & & & & \\
\hline 70 & Unidentified_92 & & & & & $v$ \\
\hline 71 & Unidentified_93 & & & & & v \\
\hline 72 & Vaccinium wisselianum & $v$ & & & & \\
\hline 73 & Ziziphus jujuba & $v$ & & & & \\
\hline & Number of species & 49 & 51 & 10 & 3 & 18 \\
\hline
\end{tabular}


Table Appendix 3. Tree species in each land cover type in Merauke District

\begin{tabular}{|c|c|c|c|c|c|c|c|c|c|c|c|}
\hline \multirow[t]{2}{*}{ No. } & \multirow[t]{2}{*}{ Tree species } & \multicolumn{10}{|c|}{ Land cover } \\
\hline & & UDLF & DDLF & USF & DSF & UM & DM & SDL & SSW & CAF & TBM \\
\hline 1 & Acacia acutifolium & & & v & & & & & & & \\
\hline 2 & Acacia auriculiformis & & v & v & $v$ & & & v & v & $v$ & \\
\hline 3 & Acacia crassicarpa & & & v & & & & & v & & \\
\hline 4 & Acacia decurrens & & v & & $v$ & & & v & & & \\
\hline 5 & Acacia leptocarpa & & v & v & v & & & & v & & \\
\hline 6 & Acacia mangium & v & v & v & $v$ & & & v & v & & \\
\hline 7 & Actinodaphne nitida & v & v & & & & & & & & \\
\hline 8 & $\begin{array}{l}\text { Adenanthera } \\
\text { microsperma }\end{array}$ & & v & & & & & & & & \\
\hline 9 & Adina sp. & v & v & & & & & & & & \\
\hline 10 & Aegialitis annulata & & & & & $v$ & & & & & \\
\hline 11 & $\begin{array}{l}\text { Aegiceras } \\
\text { corniculatum }\end{array}$ & & & & & $v$ & & & & & \\
\hline 12 & Aglaia argentea & v & v & & & & & & & & \\
\hline 13 & Aglaia cucullata & v & v & v & $v$ & & & & & & \\
\hline 14 & Aglaia sp. & & & & & & & & & $v$ & \\
\hline 15 & Aleurites moluccana & & v & & & & & & v & $v$ & v \\
\hline 16 & Alphitonia incana & & v & v & v & & & & v & & \\
\hline 17 & Alstonia actynophylla & & & v & $v$ & & & v & v & & \\
\hline 18 & Alstonia cf beatricis & v & v & v & $v$ & & & v & v & v & \\
\hline 19 & Alstonia scholaris & v & v & & & & & & & & \\
\hline 20 & $\begin{array}{l}\text { Anacardium } \\
\text { occidentale }\end{array}$ & & & & & & & & & v & v \\
\hline 21 & Anisoptera polyandra & v & & & & & & & & & \\
\hline 22 & $\begin{array}{l}\text { Anthocephalus } \\
\text { chinensis }\end{array}$ & v & & & & & & & & & \\
\hline 23 & Artocarpus fretessii & & & & & & & & & $v$ & \\
\hline 24 & $\begin{array}{l}\text { Artocarpus } \\
\text { heterophylla }\end{array}$ & & & & & & & & & $v$ & \\
\hline 25 & Artocarpus integra & & & & & & & & & $v$ & \\
\hline 26 & Artocarpus sp. & & & & & & & & & $v$ & \\
\hline 27 & $\begin{array}{l}\text { Asteromyrtus } \\
\text { symphyocarpa }\end{array}$ & & v & v & $v$ & & & & v & & \\
\hline 28 & Astronia spectabilis & & v & & & & & & & & \\
\hline 29 & $\begin{array}{l}\text { Avicennia } \\
\text { eucalyptifolia }\end{array}$ & & & & & $v$ & v & & & & \\
\hline 30 & Avicennia officinalis & & & & & $v$ & $v$ & & & & \\
\hline 31 & Baccaurea sp. & v & & & & & & & & & \\
\hline 32 & Banksia dentata & & & & $v$ & & & $v$ & & & \\
\hline 33 & $\begin{array}{l}\text { Barringtonia } \\
\text { acutangula }\end{array}$ & & & & & & & & & $v$ & \\
\hline 34 & Bruguiera cylindrica & & & & & $v$ & $v$ & & & & \\
\hline
\end{tabular}




\begin{tabular}{|c|c|c|c|c|c|c|c|c|c|c|}
\hline 35 & $\begin{array}{l}\text { Bruguiera } \\
\text { gymnorrhiza }\end{array}$ & & & & & $v$ & v & & & \\
\hline 36 & $\begin{array}{l}\text { Buchanania } \\
\text { arborescens }\end{array}$ & v & v & v & v & & & v & & \\
\hline 37 & Callistemon sp. & & & v & & & & v & & \\
\hline 38 & $\begin{array}{l}\text { Calophyllum } \\
\text { euryphyllum }\end{array}$ & v & v & & & & & v & & \\
\hline 39 & $\begin{array}{l}\text { Calophyllum } \\
\text { euryphyllum }\end{array}$ & $v$ & & & & & & & & \\
\hline 40 & $\begin{array}{l}\text { Calophyllum } \\
\text { neoebudicum }\end{array}$ & & v & & & & & & & \\
\hline 41 & $\begin{array}{l}\text { Calophyllum } \\
\text { savanarum }\end{array}$ & & v & & & & & & & \\
\hline 42 & Calophyllum soulattri & v & & & & & & v & & \\
\hline 43 & Calophyllum vexans & V & & & & & & & & \\
\hline 44 & $\begin{array}{l}\text { Canarium } \\
\text { australianum }\end{array}$ & v & & & & & & & & \\
\hline 45 & Cassia alata & & & & $\mathrm{v}$ & & & & v & v \\
\hline 46 & Ceiba pentandra & & & & & & & & & v \\
\hline 47 & Ceriops decandra & & & & & $\mathrm{v}$ & v & & & \\
\hline 48 & $\begin{array}{l}\text { Chionanthus } \\
\text { macrocarpa }\end{array}$ & & & v & & & & & & \\
\hline 49 & $\begin{array}{l}\text { Chionanthus } \\
\text { macrocarpus }\end{array}$ & & v & & & & & & & \\
\hline 50 & Citrus aurantium & & & & & & & & & v \\
\hline 51 & Citrus nobilis & & & & & & & & & v \\
\hline 52 & Cocos nucifera & & & & & & & & & v \\
\hline 53 & $\begin{array}{l}\text { Codiaeum } \\
\text { variegatum }\end{array}$ & & & & & & & & & v \\
\hline 54 & $\begin{array}{l}\text { Cryptocarya } \\
\text { densiflora }\end{array}$ & $\mathrm{v}$ & & & & & & & & \\
\hline 55 & $\begin{array}{l}\text { Cryptocarya } \\
\text { palmerensis }\end{array}$ & & v & & & & & & & \\
\hline 56 & Cryptocarya sp. & v & v & & & & & & & \\
\hline 57 & $\begin{array}{l}\text { Decaspermum } \\
\text { fruticosum }\end{array}$ & & $v$ & v & v & & & & v & \\
\hline 58 & Decaspermum sp. & & v & & & & & & & \\
\hline 59 & Dillenia alata & & & v & v & & & & & \\
\hline 60 & Dillenia papuana & & & v & V & & & v & v & \\
\hline 61 & $\begin{array}{l}\text { Dipterocarpus } \\
\text { cornutus }\end{array}$ & $\mathrm{v}$ & v & & & & & & & \\
\hline 62 & Dracontomelon edule & v & & & & & & & & \\
\hline 63 & Durio sp. & & & & & & & & & v \\
\hline 64 & Durio zibethinus & & & & & & & & & v \\
\hline 65 & $\begin{array}{l}\text { Elaeocarpus } \\
\text { arnhemicus }\end{array}$ & v & & & & & & v & & \\
\hline
\end{tabular}




\begin{tabular}{|c|c|c|c|c|c|c|c|c|c|c|c|}
\hline 66 & $\begin{array}{l}\text { Elaeocarpus cf. } \\
\text { sepikanus }\end{array}$ & v & v & & & & & & & & \\
\hline 67 & $\begin{array}{l}\text { Elaeocarpus } \\
\text { sphaericus }\end{array}$ & v & & & & & & & & & \\
\hline 68 & Erithryna sp. & & & v & & & & & v & v & \\
\hline 69 & Eucalyptus pellita & v & v & v & v & & & $\mathrm{v}$ & v & $\mathrm{v}$ & \\
\hline 70 & Eucalyptus sp. & v & v & v & & & & v & & & \\
\hline 71 & Eugenia sp. & v & v & & & & & $\mathrm{v}$ & & v & \\
\hline 72 & Eugenia sp.1 & & v & v & & & & & & & \\
\hline 73 & Eugenia suringariana & & v & & & & & & & & \\
\hline 74 & Euodia elleryana & v & v & v & & & & $\mathrm{v}$ & & & \\
\hline 75 & Excoecaria agallocha & & & & & $\mathrm{v}$ & v & & & & \\
\hline 76 & Fagraea racemosa & v & v & & & & & & & v & \\
\hline 77 & Ficus sp. 2 & & & & & & & & & v & \\
\hline 78 & Ficus septica & & & & & & & & & & v \\
\hline 79 & Ficus sp. & v & v & & v & & & & & & \\
\hline 80 & Ficus sp.1 & & & & & & & & v & & \\
\hline 81 & Flindersia laevicarpa & v & & & & & & & & & \\
\hline 82 & $\begin{array}{l}\text { Flindersia } \\
\text { pimenteliana }\end{array}$ & v & & & & & & & & & \\
\hline 83 & Garcinia dulcis & v & & v & & & & $\mathrm{v}$ & & & \\
\hline 84 & Garcinia latissima & v & & & v & & & v & & & \\
\hline 85 & Gliricidia sepium & & & & & & & & & v & \\
\hline 86 & Glochidion sp. & & v & & v & & & & v & v & v \\
\hline 87 & Gnetum gnemon & v & v & & & & & & & & \\
\hline 88 & $\begin{array}{l}\text { Gonocaryum } \\
\text { pyriforme }\end{array}$ & v & v & & & & & & & v & \\
\hline 89 & Grevillea papuana & & & & v & & & & & & \\
\hline 90 & Guettarda speciosa & & v & & & & & & & & \\
\hline 91 & Heritiera littoralis & & & & & $\mathrm{v}$ & v & & & & \\
\hline 92 & Hevea brasiliensis & & & & & & & & & & v \\
\hline 93 & Hibiscus tiliaceus & & & & & $\mathrm{v}$ & v & & & & \\
\hline 94 & Homalium foetidum & v & & & & & & & & & \\
\hline 95 & Jagera pseudorhus & & & & v & & & & v & & \\
\hline 96 & Jatropha curcas & & & & & & & & v & v & \\
\hline 97 & Knema tomentella & v & v & & & & & & & & \\
\hline 98 & Lansium domesticum & & & & & & & & & v & \\
\hline 99 & Leucaena glauca & & & v & & & & & & v & \\
\hline 100 & $\begin{array}{l}\text { Leucaena } \\
\text { leucocephala }\end{array}$ & v & & & & & & & & & \\
\hline 101 & Leucosyke sp. & & & v & & & & & & & \\
\hline 102 & Linociera lanceolata & v & & & & & & & & & \\
\hline 103 & $\begin{array}{l}\text { Lithocarpus } \\
\text { rufovillosus }\end{array}$ & $\mathrm{v}$ & & & & & & & & & \\
\hline
\end{tabular}




\begin{tabular}{|c|c|c|c|c|c|c|c|c|c|c|c|}
\hline 104 & Litsea ledermannii & $\mathrm{v}$ & & & & & & & & & \\
\hline 105 & Litsea sp & & $\mathrm{v}$ & & & & & & & & \\
\hline 106 & Litsea sp.4 & & v & & & & & & & & \\
\hline 107 & Litsea sp1 & $\mathrm{v}$ & & & & & & & & & \\
\hline 108 & Litsea sp3 & & $\mathrm{v}$ & & & & & & & & \\
\hline 109 & Litsea tuberculata & $\mathrm{v}$ & & & & & & & & & \\
\hline 110 & Lumnitzera littoralis & & & & & v & & & & & \\
\hline 111 & Lumnitzera racemosa & & & & & $v$ & $v$ & & & & \\
\hline 112 & Macaranga sp. & v & $\mathrm{v}$ & & & & & & & & \\
\hline 113 & Macaranga sp1 & $\mathrm{v}$ & & & & & & & & & \\
\hline 114 & Macaranga tanarius & v & & & & & & & $v$ & & \\
\hline 115 & Mallotus sp & & $\mathrm{v}$ & & & & & & & & \\
\hline 116 & Mangifera indica & & & & & & & & & $v$ & \\
\hline 117 & Mangifera sp. & & & & & & & & & $v$ & \\
\hline 118 & Melaleuca cajuputi & & v & $v$ & v & & & v & $v$ & $v$ & \\
\hline 119 & $\begin{array}{l}\text { Melaleuca } \\
\text { leucadendra }\end{array}$ & & $\mathrm{v}$ & v & v & & & $v$ & $v$ & & \\
\hline 120 & $\begin{array}{l}\text { Melaleuca } \\
\text { leucadendron }\end{array}$ & & & v & & & & & $v$ & & \\
\hline 121 & Melaleuca sp. & & & v & & & & & & & \\
\hline 122 & Melaleuca viridiflora & & & & & & & v & $v$ & & \\
\hline 123 & $\begin{array}{l}\text { Melastoma } \\
\text { malabathricum }\end{array}$ & & v & & v & & & & & $v$ & \\
\hline 124 & Meliosma angulata & & & & v & & & & & & \\
\hline 125 & $\begin{array}{l}\text { Metrosideros } \\
\text { petiolata }\end{array}$ & $\mathrm{v}$ & $\mathrm{v}$ & v & v & & & & $v$ & & \\
\hline 126 & Metroxylon sagu & & & & & & & & & $v$ & \\
\hline 127 & Mimusops elengi & & & & v & & & & $v$ & & \\
\hline 128 & Morinda citrifolia & & & & v & & & & v & $v$ & \\
\hline 129 & Myristica fatua & $\mathrm{v}$ & & & & & & & & & \\
\hline 130 & Myristica kajewski & & v & & & & & & & & \\
\hline 131 & Nauclea orientalis & & & v & v & & & & v & v & v \\
\hline 132 & $\begin{array}{l}\text { Nephelium } \\
\text { lappaceum }\end{array}$ & & & & & & & & & v & v \\
\hline 133 & $\begin{array}{l}\text { Palaquium } \\
\text { amboinense }\end{array}$ & $\mathrm{v}$ & $\mathrm{v}$ & & & & & & & & \\
\hline 134 & Parkia speciosa & & & & & & & & & $v$ & \\
\hline 135 & $\begin{array}{l}\text { Pimeliodendron } \\
\text { amboinicum }\end{array}$ & $\mathrm{v}$ & & & & & & & & & \\
\hline 136 & Pithecellobium & & & & & & & & & v & \\
\hline 137 & Planchonia careya & & & & $\mathrm{v}$ & & & v & & & \\
\hline 138 & Pleomele angustifolia & v & & $v$ & & & & & & & \\
\hline 139 & Podocarpus blumei & v & & & & & & & & & \\
\hline 140 & Polyalthia glauca & $v$ & & & & & & & & & \\
\hline
\end{tabular}




\begin{tabular}{|c|c|c|c|c|c|c|c|c|c|c|c|}
\hline 141 & Pongamia pinnata & & & & & v & & & & & \\
\hline 142 & Premna corymbosa & & & & & & v & & & & \\
\hline 143 & Psidium guajava & & & & & & & & & v & \\
\hline 144 & Psychotria nesophila & & & & $v$ & & & & & & \\
\hline 145 & Rhizophora apiculata & & & & & v & $v$ & & & & \\
\hline 146 & Rhizophora stylosa & & & & & v & v & & & & \\
\hline 147 & Rhodamnia cinerea & & v & v & $v$ & & & v & & & \\
\hline 148 & Semecarpus decipiens & v & & & & & & & & & \\
\hline 149 & Sonneratia acida & & & & & & $v$ & & & & \\
\hline 150 & Sonneratia alba & & & & & $v$ & & & & & \\
\hline 151 & Sterculia parkinsonii & $v$ & v & & & & & & & & \\
\hline 152 & Sterculia quadrifida & & & & $v$ & & & & & & \\
\hline 153 & Sterculia sp. & & & & & & & v & & & \\
\hline 154 & Syzygium aqueum & & & v & & & & & & $v$ & \\
\hline 155 & Syzygium cauliflorum & $\mathrm{v}$ & v & v & $v$ & & & & & & \\
\hline 156 & Syzygium fibrosum & & & & $v$ & & & & v & & \\
\hline 157 & Syzygium sp.2 & $v$ & v & v & $v$ & & & & v & & \\
\hline 158 & Syzygium sp. & & v & v & & & & v & $v$ & & \\
\hline 159 & Syzygium sp.1 & & & & $v$ & & & & & & \\
\hline 160 & $\begin{array}{l}\text { Tabernaemontana } \\
\text { pubescens }\end{array}$ & $v$ & $v$ & $v$ & $v$ & & & & & & $v$ \\
\hline 161 & Terminalia catappa & & & & & v & & & v & v & \\
\hline 162 & Timonius timon & & & & & & & & v & $v$ & \\
\hline 163 & Trema orientalis & $v$ & & & & & & & & & \\
\hline 164 & Uncaria gambir & $v$ & $v$ & & & & & & & & \\
\hline 165 & Vatica papuana & v & v & & & & & & & & \\
\hline 166 & Voacanga grandifolia & & v & & & & & & & & \\
\hline 167 & Xanthostemon brassii & & v & v & $v$ & & & v & & & \\
\hline 168 & $\begin{array}{l}\text { Xanthostemon } \\
\text { crenulatus }\end{array}$ & & & & $v$ & & & v & & & \\
\hline 169 & $\begin{array}{l}\text { Xylocarpus } \\
\text { moluccensis }\end{array}$ & & & & & v & $v$ & & & & \\
\hline & Number of species & 64 & 62 & 38 & 41 & 18 & 14 & 27 & 32 & 45 & 8 \\
\hline
\end{tabular}

Notes: UDLF = Undisturbed dry land forest; DDLF = disturbed dry land forest; USF = undisturbed swamp forest; $\mathrm{DSF}=$ disturbed swamp forest; $\mathrm{UM}=$ undisturbed mangrove; $\mathrm{DM}=$ Disturbed mangrove; $\mathrm{SDL}=$ shrub on dry land; $\mathrm{SSW}=$ shrub on swamp; $\mathrm{CAF}=$ complex agroforest; $\mathrm{TBM}=$ Treebased monoculture 


\section{WORKING PAPERS WITH DOIs}

2005

1. Agroforestry in the drylands of eastern Africa: a call to action

2. Biodiversity conservation through agroforestry: managing tree species diversity within a network of community-based, nongovernmental, governmental and research organizations in western Kenya.

3. Invasion of prosopis juliflora and local livelihoods: Case study from the Lake Baringo area of Kenya

4. Leadership for change in farmers organizations: Training report: Ridar Hotel, Kampala, 29th March to 2nd April 2005.

5. Domestication des espèces agroforestières au Sahel : situation actuelle et perspectives

6. Relevé des données de biodiversité ligneuse: Manuel du projet biodiversité des parcs agroforestiers au Sahel

7. Improved land management in the Lake Victoria Basin: TransVic Project's draft report.

8. Livelihood capital, strategies and outcomes in the Taita hills of Kenya

9. Les espèces ligneuses et leurs usages: Les préférences des paysans dans le Cercle de Ségou, au Mali

10. La biodiversité des espèces ligneuses: Diversité arborée et unités de gestion du terroir dans le Cercle de Ségou, au Mali

\section{6}

11. Bird diversity and land use on the slopes of Mt. Kilimanjaro and the adjacent plains, Tanzania

12. Water, women and local social organization in the Western Kenya Highlands

13. Highlights of ongoing research of the World Agroforestry Centre in Indonesia

14. Prospects of adoption of tree-based systems in a rural landscape and its likely impacts on carbon stocks and farmers' welfare: The FALLOW Model Application in Muara Sungkai, Lampung, Sumatra, in a 'Clean Development Mechanism' context

15. Equipping integrated natural resource managers for healthy Agroforestry landscapes.

17. Agro-biodiversity and CGIAR tree and forest science: approaches and examples from Sumatra.

18. Improving land management in eastern and southern Africa: A review of policies.

19. Farm and household economic study of Kecamatan Nanggung, Kabupaten Bogor, Indonesia: A socio-economic base line study of Agroforestry innovations and livelihood enhancement.

20. Lessons from eastern Africa's unsustainable charcoal business.

21. Evolution of RELMA's approaches to land management: Lessons from two decades of research and development in eastern and southern Africa

22. Participatory watershed management: Lessons from RELMA's work with farmers in eastern Africa.

23. Strengthening farmers' organizations: The experience of RELMA and ULAMP.

24. Promoting rainwater harvesting in eastern and southern Africa.

25. The role of livestock in integrated land management.

26. Status of carbon sequestration projects in Africa: Potential benefits and challenges to scaling up. 
27. Social and Environmental Trade-Offs in Tree Species Selection: A Methodology for Identifying Niche Incompatibilities in Agroforestry [Appears as AHI Working Paper no. 9]

28. Managing tradeoffs in agroforestry: From conflict to collaboration in natural resource management. [Appears as AHI Working Paper no. 10]

29. Essai d'analyse de la prise en compte des systemes agroforestiers pa les legislations forestieres au Sahel: Cas du Burkina Faso, du Mali, du Niger et du Senegal.

30. Etat de la recherche agroforestière au Rwanda etude bibliographique, période 1987-2003

2007

31. Science and technological innovations for improving soil fertility and management in Africa: A report for NEPAD's Science and Technology Forum.

32. Compensation and rewards for environmental services.

33. Latin American regional workshop report compensation.

34. Asia regional workshop on compensation ecosystem services.

35. Report of African regional workshop on compensation ecosystem services.

36. Exploring the inter-linkages among and between compensation and rewards for ecosystem services CRES and human well-being

37. Criteria and indicators for environmental service compensation and reward mechanisms: realistic, voluntary, conditional and pro-poor

38. The conditions for effective mechanisms of compensation and rewards for environmental services.

39. Organization and governance for fostering Pro-Poor Compensation for Environmental Services.

40. How important are different types of compensation and reward mechanisms shaping poverty and ecosystem services across Africa, Asia \& Latin America over the Next two decades?

41. Risk mitigation in contract farming: The case of poultry, cotton, woodfuel and cereals in East Africa.

42. The RELMA savings and credit experiences: Sowing the seed of sustainability

43. Yatich J., Policy and institutional context for NRM in Kenya: Challenges and opportunities for Landcare.

44. Nina-Nina Adoung Nasional di So! Field test of rapid land tenure assessment (RATA) in the Batang Toru Watershed, North Sumatera.

45. Is Hutan Tanaman Rakyat a new paradigm in community based tree planting in Indonesia?

46. Socio-Economic aspects of brackish water aquaculture (Tambak) production in Nanggroe Aceh Darrusalam.

47. Farmer livelihoods in the humid forest and moist savannah zones of Cameroon.

48. Domestication, genre et vulnérabilité : Participation des femmes, des Jeunes et des catégories les plus pauvres à la domestication des arbres agroforestiers au Cameroun.

49. Land tenure and management in the districts around Mt Elgon: An assessment presented to the Mt Elgon ecosystem conservation programme.

50. The production and marketing of leaf meal from fodder shrubs in Tanga, Tanzania: A pro-poor enterprise for improving livestock productivity.

51. Buyers Perspective on Environmental Services (ES) and Commoditization as an approach to liberate ES markets in the Philippines. 
52. Towards Towards community-driven conservation in southwest China: Reconciling state and local perceptions.

53. Biofuels in China: An Analysis of the Opportunities and Challenges of Jatropha curcas in Southwest China.

54. Jatropha curcas biodiesel production in Kenya: Economics and potential value chain development for smallholder farmers

55. Livelihoods and Forest Resources in Aceh and Nias for a Sustainable Forest Resource Management and Economic Progress

56. Agroforestry on the interface of Orangutan Conservation and Sustainable Livelihoods in Batang Toru, North Sumatra.

2008

57. Assessing Hydrological Situation of Kapuas Hulu Basin, Kapuas Hulu Regency, West Kalimantan.

58. Assessing the Hydrological Situation of Talau Watershed, Belu Regency, East Nusa Tenggara.

59. Kajian Kondisi Hidrologis DAS Talau, Kabupaten Belu, Nusa Tenggara Timur.

60. Kajian Kondisi Hidrologis DAS Kapuas Hulu, Kabupaten Kapuas Hulu, Kalimantan Barat.

61. Lessons learned from community capacity building activities to support agroforest as sustainable economic alternatives in Batang Toru orang utan habitat conservation program (Martini, Endri et al.)

62. Mainstreaming Climate Change in the Philippines.

63. A Conjoint Analysis of Farmer Preferences for Community Forestry Contracts in the Sumber Jaya Watershed, Indonesia.

64. The highlands: a shared water tower in a changing climate and changing Asia

65. Eco-Certification: Can It Deliver Conservation and Development in the Tropics.

66. Designing ecological and biodiversity sampling strategies. Towards mainstreaming climate change in grassland management.

67. Towards mainstreaming climate change in grassland management policies and practices on the Tibetan Plateau

68. An Assessment of the Potential for Carbon Finance in Rangelands

69 ECA Trade-offs Among Ecosystem Services in the Lake Victoria Basin.

69. The last remnants of mega biodiversity in West Java and Banten: an in-depth exploration of RaTA (Rapid Land Tenure Assessment) in Mount Halimun-Salak National Park Indonesia

70. Le business plan d'une petite entreprise rurale de production et de commercialisation des plants des arbres locaux. Cas de quatre pépinières rurales au Cameroun.

71. Les unités de transformation des produits forestiers non ligneux alimentaires au Cameroun. Diagnostic technique et stratégie de développement Honoré Tabuna et Ingratia Kayitavu.

72. Les exportateurs camerounais de safou (Dacryodes edulis) sur le marché sous régional et international. Profil, fonctionnement et stratégies de développement.

73. Impact of the Southeast Asian Network for Agroforestry Education (SEANAFE) on agroforestry education capacity.

74. Setting landscape conservation targets and promoting them through compatible land use in the Philippines.

75. Review of methods for researching multistrata systems. 
76. Study on economical viability of Jatropha curcas L. plantations in Northern Tanzania assessing farmers' prospects via cost-benefit analysis

77. Cooperation in Agroforestry between Ministry of Forestry of Indonesia and International Center for Research in Agroforestry

78. "China's bioenergy future. an analysis through the Lens if Yunnan Province

79. Land tenure and agricultural productivity in Africa: A comparative analysis of the economics literature and recent policy strategies and reforms

80. Boundary organizations, objects and agents: linking knowledge with action in Agroforestry watersheds

81. Reducing emissions from deforestation and forest degradation (REDD) in Indonesia: options and challenges for fair and efficient payment distribution mechanisms

2009

82. Mainstreaming climate change into agricultural education: challenges and perspectives

83. Challenging conventional mindsets and disconnects in conservation: the emerging role of ecoagriculture in Kenya's landscape mosaics

84. Lesson learned RATA garut dan bengkunat: suatu upaya membedah kebijakan pelepasan kawasan hutan dan redistribusi tanah bekas kawasan hutan

85. The emergence of forest land redistribution in Indonesia

86. Commercial opportunities for fruit in Malawi

87. Status of fruit production processing and marketing in Malawi

88. Fraud in tree science

89. Trees on farm: analysis of global extent and geographical patterns of agroforestry

90. The springs of Nyando: water, social organization and livelihoods in Western Kenya

91. Building capacity toward region-wide curriculum and teaching materials development in agroforestry education in Southeast Asia

92. Overview of biomass energy technology in rural Yunnan (Chinese - English abstract)

93. A pro-growth pathway for reducing net GHG emissions in China

94. Analysis of local livelihoods from past to present in the central Kalimantan Ex-Mega Rice Project area

95. Constraints and options to enhancing production of high quality feeds in dairy production in Kenya, Uganda and Rwanda

2010

96. Agroforestry education in the Philippines: status report from the Southeast Asian Network for Agroforestry Education (SEANAFE)

97. Economic viability of Jatropha curcas L. plantations in Northern Tanzania- assessing farmers' prospects via cost-benefit analysis.

98. Hot spot of emission and confusion: land tenure insecurity, contested policies and competing claims in the central Kalimantan Ex-Mega Rice Project area

99. Agroforestry competences and human resources needs in the Philippines

100. CES/COS/CIS paradigms for compensation and rewards to enhance environmental Services 
101. Case study approach to region-wide curriculum and teaching materials development in agroforestry education in Southeast Asia

102. Stewardship agreement to reduce emissions from deforestation and degradation (REDD): Lubuk Beringin's Hutan Desa as the first village forest in Indonesia

103. Landscape dynamics over time and space from ecological perspective

104. Komoditisasi atau koinvestasi jasa lingkungan: skema imbal jasa lingkungan program peduli sungai di DAS Way Besai, Lampung, Indonesia

105. Improving smallholders' rubber quality in Lubuk Beringin, Bungo district, Jambi province, Indonesia: an initial analysis of the financial and social benefits

106. Rapid Carbon Stock Appraisal (RACSA) in Kalahan, Nueva Vizcaya, Philippines

107. Tree domestication by ICRAF and partners in the Peruvian Amazon: lessons learned and future prospects in the domain of the Amazon Initiative eco-regional program

108. Memorias del Taller Nacional: "Iniciativas para Reducir la Deforestación en la region Andino Amazónica", 09 de Abril del 2010. Proyecto REALU Peru

109. Percepciones sobre la Equidad y Eficiencia en la cadena de valor de REDD en Perú -Reporte de Talleres en Ucayali, San Martín y Loreto, 2009. Proyecto REALU-Perú.

110. Reducción de emisiones de todos los Usos del Suelo. Reporte del Proyecto REALU Perú Fase 1

111. Programa Alternativas a la Tumba-y-Quema (ASB) en el Perú. Informe Resumen y Síntesis de la Fase II. 2da. versión revisada

112. Estudio de las cadenas de abastecimiento de germoplasma forestal en la amazonía Boliviana

113. Biodiesel in the Amazon

114. Estudio de mercado de semillas forestales en la amazonía Colombiana

115. Estudio de las cadenas de abastecimiento de germoplasma forestal en Ecuador http://dx.doi.org10.5716/WP10340.PDF

116. How can systems thinking, social capital and social network analysis help programs achieve impact at scale?

117. Energy policies, forests and local communities in the Ucayali Region, Peruvian Amazon

118. NTFPs as a Source of Livelihood Diversification for Local Communities in the Batang Toru Orangutan Conservation Program

119. Studi Biodiversitas: Apakah agroforestry mampu mengkonservasi keanekaragaman hayati di DAS Konto?

120. Estimasi Karbon Tersimpan di Lahan-lahan Pertanian di DAS Konto, Jawa Timur

121. Implementasi Kaji Cepat Hidrologi (RHA) di Hulu DAS Brantas, Jawa Timur. http://dx.doi.org/10.5716/WP10338.PDF

122. Kaji Cepat Hidrologi di Daerah Aliran Sungai Krueng Peusangan, NAD,Sumatra http://dx.doi.org/10.5716/WP10337.PDF

123. A Study of Rapid Hydrological Appraisal in the Krueng Peusangan Watershed, NAD, Sumatra. http://dx.doi.org/10.5716/WP10339.PDF

2011

124. An Assessment of farm timber value chains in Mt Kenya area, Kenya

125. A Comparative financial analysis of current land use systems and implications for the adoption of improved agroforestry in the East Usambaras, Tanzania

126. Agricultural monitoring and evaluation systems 
127. Challenges and opportunities for collaborative landscape governance in the East Usambara Mountains, Tanzania

128. Transforming Knowledge to Enhance Integrated Natural Resource Management Research, Development and Advocacy in the Highlands of Eastern Africa http://dx.doi.org/10.5716/WP11084.PDF

129. Carbon-forestry projects in the Philippines: potential and challenges The Mt Kitanglad Range forest-carbon development http://dx.doi.org10.5716/WP11054.PDF

130. Carbon forestry projects in the Philippines: potential and challenges. The Arakan Forest Corridor forest-carbon project. http://dx.doi.org10.5716/WP11055.PDF

131. Carbon-forestry projects in the Philippines: potential and challenges. The Laguna Lake Development Authority's forest-carbon development project. http://dx.doi.org/10.5716/WP11056.PDF

132. Carbon-forestry projects in the Philippines: potential and challenges. The Quirino forest-carbon development project in Sierra Madre Biodiversity Corridor http://dx.doi.org10.5716/WP11057.PDF

133. Carbon-forestry projects in the Philippines: potential and challenges. The Ikalahan Ancestral Domain forest-carbon development http://dx.doi.org10.5716/WP11058.PDF

134. The Importance of Local Traditional Institutions in the Management of Natural Resources in the Highlands of Eastern Africa. http://dx.doi.org/10.5716/WP11085.PDF

135. Socio-economic assessment of irrigation pilot projects in Rwanda. http://dx.doi.org/10.5716/WP11086.PDF

136. Performance of three rambutan varieties (Nephelium lappaceum L.) on various nursery media. http://dx.doi.org/10.5716/WP11232.PDF

137. Climate change adaptation and social protection in agroforestry systems: enhancing adaptive capacity and minimizing risk of drought in Zambia and Honduras http://dx.doi.org/10.5716/WP11269.PDF

138. Does value chain development contribute to rural poverty reduction? Evidence of asset building by smallholder coffee producers in Nicaragua http://dx.doi.org/10.5716/WP11271.PDF

139. Potential for biofuel feedstock in Kenya. http://dx.doi.org/10.5716/WP11272.PDF

140. Impact of fertilizer trees on maize production and food security in six districts of Malawi. http://dx.doi.org/10.5716/WP11281.PDF

2012

141. Fortalecimiento de capacidades para la gestión del Santuario Nacional Pampa Hermosa: Construyendo las bases para un manejo adaptativo para el desarrollo local. Memorias del Proyecto. http://dx.doi.org/10.5716/WP12005.PDF

142. Understanding rural institutional strengthening: A cross-level policy and institutional framework for sustainable development in Kenya http://dx.doi.org/10.5716/WP12012.PDF

143. Climate change vulnerability of agroforestry http://dx.doi.org/10.5716/WP16722.PDF

144. Rapid assesment of the inner Niger delta of Mali http://dx.doi.org/10.5716/WP12021.PDF

145. Designing an incentive program to reduce on-farm deforestationin the East Usambara Mountains, Tanzania http://dx.doi.org/10.5716/WP12048.PDF

146. Extent of adoption of conservation agriculture and agroforestry in Africa: the case of Tanzania, Kenya, Ghana, and Zambia http://dx.doi.org/10.5716/WP12049.PDF 
147. Policy incentives for scaling up conservation agriculture with trees in Africa: the case of Tanzania, Kenya, Ghana and Zambia http://dx.doi.org/10.5716/WP12050.PDF

148. Commoditized or co-invested environmental services? Rewards for environmental services scheme: River Care program Way Besai watershed, Lampung, Indonesia.

http://dx.doi.org/10.5716/WP12051.PDF

149. Assessment of the headwaters of the Blue Nile in Ethiopia. http://dx.doi.org/10.5716/WP12160.PDF

150. Assessment of the uThukela Watershed, Kwazaulu. http://dx.doi.org/10.5716/WP12161.PDF

151. Assessment of the Oum Zessar Watershed of Tunisia. http://dx.doi.org/10.5716/WP12162.PDF

152. Assessment of the Ruwenzori Mountains in Uganda. http://dx.doi.org/10.5716/WP12163.PDF

153. History of agroforestry research and development in Viet Nam. Analysis of research opportunities and gaps. http://dx.doi.org/10.5716/WP12052.PDF

154. REDD+ in Indonesia: a Historical Perspective. http://dx.doi.org/10.5716/WP12053.PDF

155. Agroforestry and Forestry in Sulawesi series: Livelihood strategies and land use system dynamics in South Sulawesi http://dx.doi.org/10.5716/WP12054.PDF

156. Agroforestry and Forestry in Sulawesi series: Livelihood strategies and land use system dynamics in Southeast Sulawesi. http://dx.doi.org/10.5716/WP12055.PDF

157. Agroforestry and Forestry in Sulawesi series: Profitability and land-use systems in South and Southeast Sulawesi. http://dx.doi.org/10.5716/WP12056.PDF

158. Agroforestry and Forestry in Sulawesi series: Gender, livelihoods and land in South and Southeast Sulawesi http://dx.doi.org/10.5716/WP12057.PDF

159. Agroforestry and Forestry in Sulawesi series: Agroforestry extension needs at the community level in AgFor project sites in South and Southeast Sulawesi, Indonesia. http://dx.doi.org/10.5716/WP12058.PDF

160. Agroforestry and Forestry in Sulawesi series: Rapid market appraisal of agricultural, plantation and forestry commodities in South and Southeast Sulawesi.

http://dx.doi.org/10.5716/WP12059.PDF

2013

161. Diagnosis of farming systems in the Agroforestry for Livelihoods of Smallholder farmers in Northwestern Viet Nam project http://dx.doi.org/10.5716/WP13033.PDF

162. Ecosystem vulnerability to climate change: a literature review. http://dx.doi.org/10.5716/WP13034.PDF

163. Local capacity for implementing payments for environmental services schemes: lessons from the RUPES project in northeastern Viet Nam http://dx.doi.org/10.5716/WP13046.PDF

164. Seri Agroforestri dan Kehutanan di Sulawesi: Agroforestry dan Kehutanan di Sulawesi: Strategi mata pencaharian dan dinamika sistem penggunaan lahan di Sulawesi Selatan http://dx.doi.org/10.5716/WP13040.PDF

165. Seri Agroforestri dan Kehutanan di Sulawesi: Mata pencaharian dan dinamika sistem penggunaan lahan di Sulawesi Tenggara http://dx.doi.org/10.5716/WP13041.PDF

166. Seri Agroforestri dan Kehutanan di Sulawesi: Profitabilitas sistem penggunaan lahan di Sulawesi Selatan dan Sulawesi Tenggara http://dx.doi.org/10.5716/WP13042.PDF

167. Seri Agroforestri dan Kehutanan di Sulawesi: Gender, mata pencarian dan lahan di Sulawesi Selatan dan Sulawesi Tenggara http://dx.doi.org/10.5716/WP13043.PDF 
168. Seri Agroforestri dan Kehutanan di Sulawesi: Kebutuhan penyuluhan agroforestri pada tingkat masyarakat di lokasi proyek AgFor di Sulawesi Selatan dan Tenggara, Indonesia. http://dx.doi.org/10.5716/WP13044.PDF

169. Seri Agroforestri dan Kehutanan di Sulawesi: Laporan hasil penilaian cepat untuk komoditas pertanian, perkebunan dan kehutanan di Sulawesi Selatan dan Tenggara http://dx.doi.org/10.5716/WP13045.PDF

170. Agroforestry, food and nutritional security http://dx.doi.org/10.5716/WP13054.PDF

171. Stakeholder Preferences over Rewards for Ecosystem Services: Implications for a REDD+ Benefit Distribution System in Viet Nam http://dx.doi.org/10.5716/WP13057.PDF

172. Payments for ecosystem services schemes: project-level insights on benefits for ecosystems and the rural poor http://dx.doi.org/10.5716/WP13001.PDF

173. Good practices for smallholder teak plantations: keys to success http://dx.doi.org/10.5716/WP13246.PDF

174. Market analysis of selected agroforestry products in the Vision for Change Project intervention Zone, Côte d'Ivoire http://dx.doi.org/10.5716/WP13249.PDF

175. Rattan futures in Katingan: why do smallholders abandon or keep their gardens in Indonesia's 'rattan district'? http://dx.doi.org/10.5716/WP13251.PDF

176. Management along a gradient: the case of Southeast Sulawesi's cacao production landscapes http://dx.doi.org/10.5716/WP13265.PDF

2014

177. Are trees buffering ecosystems and livelihoods in agricultural landscapes of the Lower Mekong Basin? Consequences for climate-change adaptation. http://dx.doi.org/10.5716/WP14047.PDF

178. Agroforestry, livestock, fodder production and climate change adaptation and mitigation in East Africa: issues and options. http://dx.doi.org/10.5716/WP14050.PDF

179. Trees on farms: an update and reanalysis of agroforestry's global extent and socio-ecological characteristics. http://dx.doi.org/10.5716/WP14064.PDF

180. Beyond reforestation: an assessment of Vietnam's REDD+ readiness. http://dx.doi.org/10.5716/WP14097.PDF

181. Farmer-to-farmer extension in Kenya: the perspectives of organizations using the approach. http://dx.doi.org/10.5716/WP14380.PDF

182. Farmer-to-farmer extension in Cameroon: a survey of extension organizations. http://dx.doi.org/10.5716/WP14383.PDF

183. Farmer-to-farmer extension approach in Malawi: a survey of organizations: a survey of organizations http://dx.doi.org/10.5716/WP14391.PDF

184. Seri Agroforestri dan Kehutanan di Sulawesi: Kuantifikasi jasa lingkungan air dan karbon pola agroforestri pada hutan rakyat di wilayah sungai Jeneberang

185. Options for Climate-Smart Agriculture at Kaptumo Site in Kenyahttp://dx.doi.org/10.5716/WP14394.PDF

2015

186. Agroforestry for Landscape Restoration and Livelihood Development in Central Asia http://dx.doi.org/10.5716/WP14143.PDF 
187. "Projected Climate Change and Impact on Bioclimatic Conditions in the Central and SouthCentral Asia Region" http://dx.doi.org/10.5716/WP14144.PDF

188. Land Cover Changes, Forest Loss and Degradation in Kutai Barat, Indonesia. http://dx.doi.org/10.5716/WP14145.PDF

189. The Farmer-to-Farmer Extension Approach in Malawi: A Survey of Lead Farmers. http://dx.doi.org/10.5716/WP14152.PDF

190. Evaluating indicators of land degradation and targeting agroforestry interventions in smallholder farming systems in Ethiopia. http://dx.doi.org/10.5716/WP14252.PDF

191. Land health surveillance for identifying land constraints and targeting land management options in smallholder farming systems in Western Cameroon

192. Land health surveillance in four agroecologies in Malawi

193. Cocoa Land Health Surveillance: an evidence-based approach to sustainable management of cocoa landscapes in the Nawa region, South-West Côte d'Ivoire http://dx.doi.org/10.5716/WP14255.PDF

194. Situational analysis report: Xishuangbanna autonomous Dai Prefecture, Yunnan Province, China. http://dx.doi.org/10.5716/WP14255.PDF

195. Farmer-to-farmer extension: a survey of lead farmers in Cameroon. http://dx.doi.org/10.5716/WP15009.PDF

196. From transition fuel to viable energy source Improving sustainability in the sub-Saharan charcoal sector http://dx.doi.org/10.5716/WP15011.PDF

197. Mobilizing Hybrid Knowledge for More Effective Water Governance in the Asian Highlands http://dx.doi.org/10.5716/WP15012.PDF

198. Water Governance in the Asian Highlands http://dx.doi.org/10.5716/WP15013.PDF

199. Assessing the Effectiveness of the Volunteer Farmer Trainer Approach in Dissemination of Livestock Feed Technologies in Kenya vis-à-vis other Information Sources http://dx.doi.org/10.5716/WP15022.PDF

200. The rooted pedon in a dynamic multifunctional landscape: Soil science at the World Agroforestry Centre http://dx.doi.org/10.5716/WP15023.PDF

201. Characterising agro-ecological zones with local knowledge. Case study: Huong Khe district, Ha Tinh, Viet Nam http://dx.doi.org/10.5716/WP15050.PDF

202. Looking back to look ahead: Insight into the effectiveness and efficiency of selected advisory approaches in the dissemination of agricultural technologies indicative of Conservation Agriculture with Trees in Machakos County, Kenya. http://dx.doi.org/10.5716/WP15065.PDF

203. Pro-poor Biocarbon Projects in Eastern Africa Economic and Institutional Lessons. http://dx.doi.org/10.5716/WP15022.PDF

204. Projected climate change impacts on climatic suitability and geographical distribution of banana and coffee plantations in Nepal. http://dx.doi.org/10.5716/WP15294.PDF

205. Agroforestry and Forestry in Sulawesi series: Smallholders' coffee production and marketing in Indonesia. A case study of two villages in South Sulawesi Province. http://dx.doi.org/10.5716/WP15690.PDF

206. Mobile phone ownership and use of short message service by farmer trainers: a case study of Olkalou and Kaptumo in Kenya http://dx.doi.org/10.5716/WP15691.PDF

207. Associating multivariate climatic descriptors with cereal yields: a case study of Southern Burkina Faso http://dx.doi.org/10.5716/WP15273.PDF

208. Preferences and adoption of livestock feed practices among farmers in dairy management groups in Kenya http://dx.doi.org/10.5716/WP15675.PDF 
209. Scaling up climate-smart agriculture: lessons learned from South Asia and pathways for success http://dx.doi.org/10.5716/WP15720.PDF

210. Agroforestry and Forestry in Sulawesi series: Local perceptions of forest ecosystem services and collaborative formulation of reward mechanisms in South and Southeast Sulawesi http://dx.doi.org/10.5716/WP15721.PDF

211. Potential and challenges in implementing the co-investment of ecosystem services scheme in Buol District, Indonesia. http://dx.doi.org/10.5716/WP15722.PDF

212. Tree diversity and its utilization by the local community in Buol District, Indonesia http://dx.doi.org/10.5716/WP15723.PDF

213 Vulnerability of smallholder farmers and their preferences on farming practices in Buol District, Indonesia http://dx.doi.org/10.5716/WP15724.PDF

214. Dynamics of Land Use/Cover Change and Carbon Emission in Buol District, Indonesia http://dx.doi.org/10.5716/WP15725.PDF

215. Gender perspective in smallholder farming practices in Lantapan, Phillippines. http://dx.doi.org/10.5716/WP15726.PDF

216. Vulnerability of smallholder farmers in Lantapan, Bukidnon. http://dx.doi.org/10.5716/WP15727.PDF

217. Vulnerability and adaptive capacity of smallholder farmers in Ho Ho Sub-watershed, Ha Tinh Province, Vietnam http://dx.doi.org/10.5716/WP15728.PDF

218. Local Knowledge on the role of trees to enhance livelihoods and ecosystem services in northern central Vietnam http://dx.doi.org/10.5716/WP15729.PDF

219. Land-use/cover change in Ho Ho Sub-watershed, Ha Tinh Province, Vietnam. http://dx.doi.org/10.5716/WP15730.PDF

2016

220. Agroforestry and Forestry in Sulawesi series: Evaluation of the Agroforestry Farmer Field Schools on agroforestry management in South and Southeast Sulawesi, Indonesia. http://dx.doi.org/10.5716/WP16002.PDF

221. Farmer-to-farmer extension of livestock feed technologies in Rwanda: A survey of volunteer farmer trainers and organizations. http://dx.doi.org/10.5716/WP16005.PDF

222. Projected Climate Change Impact on Hydrology, Bioclimatic Conditions, and Terrestrial Ecosystems in the Asian Highlands http://dx.doi.org/10.5716/WP16006.PDF

223. Adoption of Agroforestry and its impact on household food security among farmers in Malawi http://dx.doi.org/10.5716/WP16013.PDF

224. Agroforestry and Forestry in Sulawesi series: Information channels for disseminating innovative agroforestry practices to villages in Southern Sulawesi, Indonesia http://dx.doi.org/10.5716/WP16034.PDF

225. Agroforestry and Forestry in Sulawesi series: Unravelling rural migration networks.Landtenure arrangements among Bugis migrant communities in Southeast Sulawesi. http://dx.doi.org/10.5716/WP16035.PDF

226. Agroforestry and Forestry in Sulawesi series: Women's participation in agroforestry: more benefit or burden? A gendered analysis of Gorontalo Province. http://dx.doi.org/10.5716/WP16036.PDF

227. Kajian Kelayakan dan Pengembangan Desain Teknis Rehabilitasi Pesisir di Sulawesi Tengah. http://dx.doi.org/10.5716/WP16037.PDF

228. Selection of son tra clones in North West Vietnam. http://dx.doi.org/10.5716/WP16038.PDF 
229. Growth and fruit yield of seedlings, cuttings and grafts from selected son tra trees in Northwest Vietnam http://dx.doi.org/10.5716/WP16046.PDF

230. Gender-Focused Analysis of Poverty and Vulnerability in Yunnan, China http://dx.doi.org/10.5716/WP16071.PDF

231. Seri Agroforestri dan Kehutanan di Sulawesi: Kebutuhan Penyuluhan Agroforestri untuk Rehabilitasi Lahan di Sumba Timur, Nusa Tenggara Timur, Indonesia. http://dx.doi.org/10.5716/WP16077.PDF

232. Agroforestry and Forestry in Sulawesi series: Agroforestry extension needs for land rehabilitation in East Sumba, East Nusa Tenggara, Indonesia. http://dx.doi.org/10.5716/WP16078.PDF

233. Central hypotheses for the third agroforestry paradigm within a common definition. http://dx.doi.org/10.5716/WP16079.PDF

234. Assessing smallholder farmers' interest in shade coffee trees: The Farming Systems of Smallholder Coffee Producers in the Gisenyi Area, Rwanda: a participatory diagnostic study. http://dx.doi.org/10.5716/WP16104.PDF

235. Review of agricultural market information systems in |sub-Saharan Africa. http://dx.doi.org/10.5716/WP16110.PDF

236. Vision and road map for establishment of a protected area in Lag Badana, Lower Jubba, Somalia. http://dx.doi.org/10.5716/WP16127.PDF

237. Replicable tools and frameworks for Bio-Carbon Development in West Africa. http://dx.doi.org/10.5716/WP16138.PDF

238. Existing Conditions, Challenges and Needs in the Implementation of Forestry and Agroforestry Extension in Indonesia. http://dx.doi.org/10.5716/WP16141.PDF

239. Situasi Terkini, Tantangan dan Kebutuhan Pelaksanaan Penyuluhan Kehutanan dan Agroforestri di Indonesia. http://dx.doi.org/10.5716/WP16142.PDF

240. The national agroforestry policy of India: experiential learning in development and delivery phases. http://dx.doi.org/10.5716/WP16143.PDF

241. Agroforestry and Forestry in Sulawesi series: Livelihood strategies and land-use system dynamics in Gorontalo. http://dx.doi.org/10.5716/WP16157.PDF

242. Seri Agroforestri dan Kehutanan di Sulawesi: Strategi mata pencaharian dan dinamika sistem penggunaan lahan di Gorontalo. http://dx.doi.org/10.5716/WP16158.PDF

243. Ruang, Gender dan Kualitas Hidup Manusia: Sebuah studi Gender pada komunitas perantau dan pengelola kebun di Jawa Barat. http://dx.doi.org/10.5716/WP16159.PDF

244. Gendered Knowledge and perception in managing grassland areas in East Sumba, Indonesia. http://dx.doi.org/10.5716/WP16160.PDF

245. Pengetahuan dan persepsi masyarakat pengelola padang aavana, Sebuah Kajian Gender di Sumba Timur. http://dx.doi.org/10.5716/WP16161.PDF

246. Dinamika Pengambilan Keputusan pada komunitas perantau dan pengelola kebun di Jawa Barat. http://dx.doi.org/10.5716/WP16162.PDF

247. Gaharu (eaglewood) domestication: Biotechnology, markets and agroforestry options. http://dx.doi.org/10.5716/WP16163.PDF

248. Marine habitats of the Lamu-Kiunga coast: an assessment of biodiversity value, threats and opportunities. http://dx.doi.org/10.5716/WP16167.PDF

249. Assessment of the biodiversity in terrestrial landscapes of the Witu protected area and surroundings, Lamu County Kenya. http://dx.doi.org/10.5716/WP16172.PDF

250. An ecosystem services perspective on benefits that people derive from biodiversity of Coastal forests in Lamu County, Kenya http://dx.doi.org/10.5716/WP16173.PDF 
251. Assessment of the biodiversity in terrestrial and marine landscapes of the proposed Laga Badana National Park and surrounding areas, Jubaland, Somalia. http://dx.doi.org/10.5716/WP16174.PDF

2017

252. Preferensi Petani terhadap Topik Penyuluhan dan Penyebaran Informasi Agroforestri di Indonesia. http://dx.doi.org/10.5716/WP16181.PDF

253. Seri Agroforestri dan Kehutanan di Sulawesi: Keanekaragaman hayati jenis pohon pada hutan rakyat agroforestri di DAS Balangtieng, Sulawesi Selatan. http://dx.doi.org/10.5716/WP16182.PDF

254. Potensi dan Tantangan dalam Pengembangan Skema Ko-Investasi Jasa Lingkungan di Kabupaten Buol, Indonesia. http://dx.doi.org/10.5716/WP17008.PDF

255. Keragaman Jenis Pohon dan Pemanfaatannya oleh Masyarakat di Kabupaten Buol, Indonesia. http://dx.doi.org/10.5716/WP17009.PDF

256. Kerentanan dan preferensi sistem pertanian petani di Kabupaten Buol, Indonesia. http://dx.doi.org/10.5716/WP17010.PDF

257. Dinamika Perubahan Penggunaan/Tutupan Lahan Serta Cadangan Karbon di Kabupaten Buol, Indonesia. http://dx.doi.org/10.5716/WP17011.PDF

258. The Effectiveness of the Volunteer Farmer Trainer Approach vis-à-vis Other Information Sources in Dissemination of Livestock Feed Technologies in Uganda. http://dx.doi.org/10.5716/WP17104.PDF

259. Agroforestry and Forestry in Sulawesi series: Impact of agricultural-extension booklets on community livelihoods in South and Southeast Sulawesi. http://dx.doi.org/10.5716/WP17125.PDF

260. Petani Menjadi Penyuluh, Mungkinkah? Sebuah Pendekatan Penyuluhan dari Petani ke Petani di Kabupaten Sumba Timur. http://dx.doi.org/10.5716/WP17145.PDF

261. Dampak Perubahan Tutupan Lahan terhadap Kondisi Hidrologi di Das Buol, Kabupaten Buol, Sulawesi Tengah: Simulasi dengan Model Genriver. http://dx.doi.org/10.5716/WP17146.PDF

262. Analisis Tapak Mata Air Umbulan, Pasuruan, Jawa Timur. Kajian elemen biofisik dan persepsi masyarakat. http://dx.doi.org/10.5716/WP17147.PDF

263. Planned comparisons demystified. http://dx.doi.org/10.5716/WP17354.PDF

264. Soil health decision support for NERC digital soil platforms: A survey report. http://dx.doi.org/10.5716/WP17355.PDF

265. Seri Pembangunan Ekonomi Pedesaan Indonesia: Menanam di bukit gundul: Pengetahuan masyarakat lokal dalam upaya restorasi lahan di Sumba Timur. http://dx.doi.org/10.5716/WP17356.PDF

266. Tree diversity and carbon stock in three districts of Kutai Timur, Pasir and Berau, East Kalimantan http://dx.doi.org/10.5716/WP17357.PDF

267. Tree Diversity and Carbon Stock in Various Land Use Systems of Banyuasin and Musi Banyuasin Districts, South Sumatera http://dx.doi.org/10.5716/WP17358.PDF

268. Tree diversity and carbon stock in various land cover systems of Jayapura, Jayawijaya and Merauke Districts, Papua Province http://dx.doi.org/10.5716/WP17359.PDF 

The World Agroforestry Centre is an autonomous, non-profit research organization whose vision is a rural transformation in the developing world as smallholder households increase their use of trees in agricultural landscapes to improve food security, nutrition, income, health, shelter, social cohesion, energy resources and environmental sustainability. The Centre generates science-based knowledge about the diverse roles that trees play in agricultural landscapes, and uses its research to advance policies and practices, and their implementation that benefit the poor and the environment. It aims to ensure that all this is achieved by enhancing the quality of its science work, increasing operational efficiency, building and maintaining strong partnerships, accelerating the use and impact of its research, and promoting greater cohesion, interdependence and alignment within the organization.

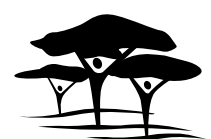

United Nations Avenue, Gigiri • PO Box 30677 • Nairobi, $00100 \cdot$ Kenya Telephone: +254 207224000 or via USA +1 6508336645 Fax: +254207224001 or via USA +1 6508336646

Email: worldagroforestry@cgiar.org•www.worldagroforestry.org

Southeast Asia Regional Program • Sindang Barang • Bogor 16680

PO Box $161 \cdot$ Bogor $16001 \cdot$ Indonesia

Telephone: +62 2518625415 • Fax: +62 2518625416

- Email: icraf-indonesia@cgiar.org

www.worldagroforestry.org/region/southeast-asia

blog.worldagroforestry.org 